\title{
A household model of German cockroach infestations and their effects on symptoms of atopic asthma
}

\author{
Amandeep Kaur ${ }^{\mathrm{a}}$, Karen Funderburk ${ }^{\mathrm{b}}$, Ana Lucía Campaña ${ }^{\mathrm{c}}$, Patricia Puente ${ }^{\mathrm{d}}$ and \\ Karen R. Ríos-Soto ${ }^{\mathrm{e}}$ \\ ${ }^{a}$ Department of Mathematics, California State University, Bakersfield, CA, USA; ${ }^{b}$ Simon A. Levin Mathematical, \\ Computational, and Modeling Sciences Center, Arizona State University, Tempe, Arizona, USA; ${ }^{\circ}$ Department \\ of Electrical and Electronic Engineering, Universidad de Los Andes, Bogotá D.C., Colombia; ${ }^{\mathrm{d}}$ Department of \\ Mathematics \& Computer Science, Texas Woman's University, Denton, Texas, USA; ${ }^{\mathrm{C}}$ Department of \\ Mathematical Sciences, University of Puerto Rico at Mayagüez, Mayagüez, Puerto Rico, USA
}

\begin{abstract}
Asthma is a chronic respiratory condition which affects 25 million people in the United States. Improper extermination of cockroaches and their associated allergens significantly increases the occurrence of atopic asthma, a type of asthma stemming from exposure to allergens. To date, dynamical studies of cockroach infestations and their effects on atopic asthma have not been examined. Here we develop a novel mathematical model, with households as the model unit, to study the dynamics of cockroach infestations within a neighbourhood and how they impact the prevalence of asthma. The focus of this study is to evaluate the impact of extermination and removal of allergens in a household containing an individual with recurrent atopic asthma. Through analysing our model, the basic reproduction number for cockroach infestations was calculated and we were able to determine conditions under which cockroaches and asthma can be eliminated from a community containing infested houses. From the presence of a backward bifurcation, we are able to show that it is more effective to prevent infestation of cockroaches than attempting to remove them post-infestation. These conclusions could potentially assist in educating the general public about the importance of pest control and its relationship with asthma.
\end{abstract}

\section{ARTICLE HISTORY}

Received 6 January 2019

Accepted 30 September 2019

\section{KEYWORDS}

Asthma; cockroach; German cockroach; mathematical model; allergens; infestation

\section{Introduction}

It is estimated that asthma affects nearly 300 million people worldwide; and by 2025 this number is expected to increase to 400 million due to an increase in atopic sensitization (Braman, 2006). Atopic asthma, the most common form of asthma, is developed from continuous exposure to allergens, such as indoor and outdoor pollution, followed by damaging allergic reactions (Ober \& Yao, 2011). This chronic condition annually takes the life of approximately 180,000 people, many of which could be avoided with proper treatment and care. Individuals with this condition present a chronic inflammation in the lower

CONTACT Karen R. Ríos-Soto karen.rios3@upr.edu 
airways that, in the proximity of common allergens, results in variable airflow obstruction, causing recurrent episodes of coughing, wheezing, breathlessness, and chest tightness (Braman, 2006).

The German cockroach (Blatella germanica), is the most likely to be found indoors, compared to other species such as the American cockroach (Periplaneta americana) or the Oriental cockroach (Blatta orientalis) (Rust, Owens, \& Reierson, 1995). Levels of cockroach allergen in homes have been studied for years, as this is a common risk factor for allergic sensitization and asthma morbidity (Arruda et al., 2001; Rosenstreich et al., 1997; Sporik et al., 1999). Because of their high reproduction rates and frequent cohabitation with humans, household infestations by cockroaches are a familiar problem. Therefore, once a cockroach enters a home, it is essential to get rid of it quickly. Cockroaches have been reported to be associated with atopic asthma in many regions of the world due to constant exposure to cockroach allergens (Call, Smith, Morris, Chapman, \& PlattsMills, 1992). Bla g 1 and Bla g 2 are the major cockroach allergens associated with the German cockroach and the highest concentrations are found in their secretions and fecal material (Cornwell, 1968). Exposure to these allergens have been reported to be among the most important risk factors in asthma morbidity and mortality for children from lowincome families living in inner cities, such as New York (Arruda et al., 2001; Wang, Abou El-Nour, \& Bennett, 2008). Strategies for decreasing environmental exposure to cockroach allergen in inner-city homes have been investigated and the results suggest that a sustained decrease in cockroach allergen levels is difficult to accomplish, even after successful extermination of cockroach populations (Arruda et al., 2001).

In a national survey of U.S. homes, $13 \%$ had a concentration higher than $2.0 \mathrm{U} / \mathrm{g}$ of the cockroach allergen Bla g 1 , which is a concentration related to the prevalence of allergic and asthmatic symptoms (Wang et al., 2008). The number of children with positive skin test responses to cockroach allergen increases rapidly as the intensity of exposure to Bla $g$ 1 increases, and some studies propose the value of $8.0 \mathrm{U} / \mathrm{g}$ as a disease-induction threshold (Eggleston \& Arruda, 2001; Eggleston et al., 1998; Rosenstreich et al., 1997).

Currently, it is very difficult to achieve complete elimination of cockroach allergens in infested homes. However, it has been shown that reductions up to $90 \%$ can be achieved through the use of an assortment of chemicals (Wood, Eggleston, Rand, Nixon, \& Kanchanaraksa, 2001). It is believed that successful reduction of cockroach allergen is also linked to the reduction of cockroach-associated asthma morbidity (Bassirpour \& Zoratti, 2014).

After a careful review of the literature, we were unable to find any dynamical studies related to cockroach infestations and their effect on atopic asthma. Therefore, the goal of this work is to study how extermination of Blattella germanica and removal of their allergens prevent the recurrence of atopic asthma symptoms in a household using a mathematical compartment model with households as the epidemiological unit. Outcomes of performing mathematical analysis, running numerical simulations and carrying out sensitivity analysis will aid in understanding this relationship.

In Section 2, we define a dynamical model describing the flow of cockroach infestations as a disease throughout a neighbourhood, with asthma attacks being the main symptom. In addition, we also include the mathematical analysis of three different subcases of the model. In Section 3, we report results from bifurcation analysis, numerical simulations and sensitivity analysis of the model. From these analyses we find that $R_{0}<1$ is not sufficient to guarantee that the threat of future asthma attacks will be eliminated within the population, 
and that prevention of infestations is the more feasible option. Our conclusions are further discussed in Section 4.

\section{Methods}

\subsection{Definition of the model}

To understand the impact of cockroaches on the incidence of asthma symptoms in a neighbourhood, we consider a mathematical compartment model, similar to the one introduced by Kermack and McKendrick (1927), describing the spread of cockroach infestations within a neighbourhood as a disease, and asthma attacks as the resulting symptoms. In this model, households are considered as the epidemiological unit, as opposed to individuals in a typical SIR model. Because the mechanism by which asthma develops is not yet fully understood, we assume that all households within the population contain at least one individual that has been sensitized to the cockroach allergens and has been previously diagnosed with asthma. For simplicity, we also assume that the number of households and the number of individuals occupying a household is constant, and that individuals do not relocate.

The model consists of four compartments (listed in Table 1): susceptible $(S(t))$, asymptomatic infested $(A(t))$, symptomatic infested $(I(t))$ and cleaned $(C(t))$ households, at time $t$. The susceptible households do not contain cockroaches or cockroach allergens, therefore no individuals in the household express asthmatic symptoms. Once a household becomes infested with cockroaches, it enters the asymptomatic infested compartment. That household is now able to spread the infestation to other homes, but individuals within the house have not yet developed asthma symptoms. Upon infestation of cockroaches and entrance into the asymptomatic infested class, that home begins to accumulate cockroach allergens.

A concentration of Bla g 1 greater than $8 \mathrm{U} / \mathrm{g}$ has been associated with greater asthma morbidity among sensitive children (Call et al., 1992; Gelber et al., 1993; Rosenstreich et al., 1997). Therefore, given the assumption that at least one individual within the home has previously been sensitized to the cockroach allergen and diagnosed with asthma, we assume that once a household reaches this threshold, that individual will undoubtedly begin to show asthma symptoms. That household then changes status to a symptomatic infested household; that is, with both cockroaches and asthma symptoms. Afterwards, successful professional extermination of the cockroaches will convert that household's status to cleaned. A cleaned household contains no cockroaches, but allergens are still present, so individuals still exhibit asthma symptoms. Afterwards, the cleaned household will either return to the symptomatic infested class or to the susceptible class. The former will occur when a cleaned household becomes reinfested with cockroaches. The latter is a result of the level of cockroach allergens in the home receding below the asthma morbidity threshold

Table 1. State Variables of Household Model.

\begin{tabular}{ll}
\hline Variable & \multicolumn{1}{c}{ Definition } \\
\hline$S(t)$ & Number of households free of cockroaches and allergens at time $t$ \\
$A(t)$ & Number of households infested with cockroaches but symptoms of asthma are not yet present at time $t$ \\
$I(t)$ & Number of households infested with cockroaches and presenting asthma symptoms at time $t$ \\
$C(t)$ & Number of houses without cockroaches but allergens and asthma symptoms still present at time $t$ \\
\hline
\end{tabular}




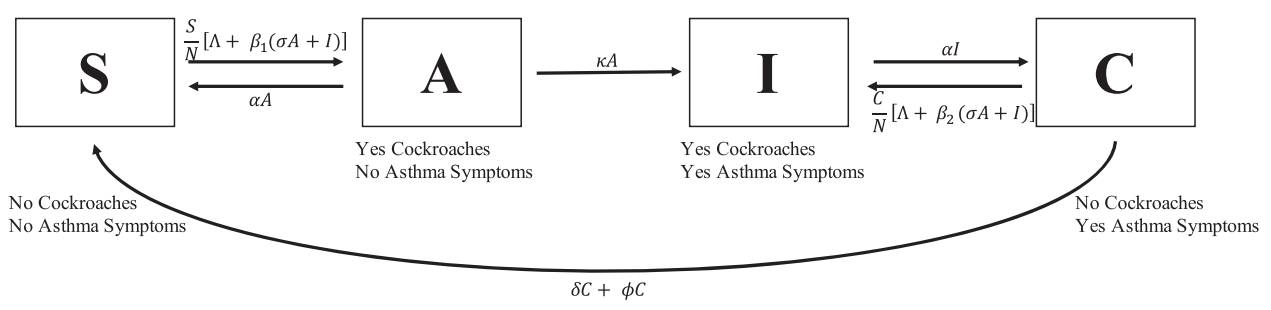

Figure 1. Schematic diagram for the household infestation stages with cockroaches, including compartments for susceptible $(S)$, asymptomatic infested $(A)$, symptomatic infested $(I)$ and Cleaned $(C)$.

of $8 \mathrm{U} / \mathrm{g}$, which we assume will eliminate the presence of asthma symptoms. Figure 1 gives a compartmental diagram representation of our model.

Because we assume a constant population, the total population at any time $t$ is represented by $N_{0}=S(t)+A(t)+I(t)+C(t)$, where $N_{0}$ is the initial number of households.

A susceptible home changes its status by becoming infested with cockroaches, which could either happen by cockroaches migrating in from outside of the neighbourhood and selecting a susceptible house at a rate $(S \Lambda / N)$, or by cockroaches coming in from other infested households at a rate $(S / N) \beta_{1}(\sigma A+I)$, where $\beta_{1}$ represents the migration rate and $\sigma$ accounts for the reduced number of cockroaches in asymptomatic infested homes compared to symptomatic infested homes. Once a household becomes infested, the cockroaches can be exterminated before symptoms are present in the household at rate $\alpha$, and return the household to susceptible status. On the other hand, if cockroaches are not exterminated, asymptomatic infected households become infested and asthmatic households at rate $\kappa$, where $1 / \kappa$ is defined to be the time that it takes for a home to build up enough allergens to cross the asthma morbidity threshold. Once the asthma morbidity threshold is crossed, we assume that it is no longer possible to reduce the level of allergens without first exterminating the cockroaches, in which case, symptomatic infested households will become cleaned households. This occurs at a rate $\alpha$. Finally, households in the cleaned compartment either become reinfested with cockroaches, or the allergens are completely eradicated. Reinfestation occurs when cockroaches migrate from outside of the neighbourhood and select a cleaned home at a rate $(C \Lambda / N)$, or migrate from infested households at rate $C \beta_{2}(\sigma A+I) / N$, where $\beta_{2}$ is the migration rate of cockroaches to houses that still contain allergens. Because cockroaches are typically attracted to places with preexisting cockroach allergens, we assume $\beta_{2}>\beta_{1}$ (Bassirpour \& Zoratti, 2014). Eradication of allergens occurs either from the professional removal of allergens, at a rate $\delta$, or from simply waiting for the potency of the allergens to dissipate with time, at a rate $\phi$. Because we assume that the cleaning is effective, $\delta>\phi$. The following system of non-linear ordinary differential equations represents the dynamics of the population of households in our model and a summary of the parameter definitions is given in Table 2.

$$
\begin{aligned}
\frac{\mathrm{d} S}{\mathrm{~d} t} & =-\frac{S}{N}\left[\Lambda+\beta_{1}(\sigma A+I)\right]+\alpha A+C(\delta+\phi), \\
\frac{\mathrm{d} A}{\mathrm{~d} t} & =-\kappa A-\alpha A+\frac{S}{N}\left[\Lambda+\beta_{1}(\sigma A+I)\right],
\end{aligned}
$$


Table 2. Parameter Definitions of Household Model.

\begin{tabular}{lll}
\hline Parameter & \multicolumn{1}{c}{ Definition } & \multicolumn{1}{c}{ Unit } \\
\hline$\Lambda$ & Influx rate of cockroaches from outside neighbourhoods & households $\cdot$ months $^{-1}$ \\
$\beta_{1}$ & Rate of cockroach infestation/migration to susceptible houses from outside & months $^{-1}$ \\
& the neighbourhood & \\
$\beta_{2}$ & Rate of cockroach reinfestation/migration to cleaned houses \\
$\sigma$ & Reduction infestation factor for asymptomatic infested houses & months $^{-1}$ \\
$\alpha$ & Extermination rate & months $^{-1}$ \\
$\delta$ & Allergen removal rate & months $^{-1}$ \\
$\phi$ & Rate of natural removal of allergen & months $^{-1}$ \\
$\kappa$ & Cockroach reproduction rate & months $^{-1}$ \\
\hline
\end{tabular}

$$
\begin{aligned}
\frac{\mathrm{d} I}{\mathrm{~d} t} & =-\alpha I+\kappa A+\frac{C}{N}\left[\Lambda+\beta_{2}(\sigma A+I)\right], \\
\frac{\mathrm{d} C}{\mathrm{~d} t} & =-(\delta+\phi) C-\frac{C}{N}\left[\Lambda+\beta_{2}(\sigma A+I)\right]+\alpha I,
\end{aligned}
$$

where $N(t)=S(t)+A(t)+I(t)+C(t)$.

This model will be studied under three different conditions. The first case $(\Lambda=0$ and $\beta_{2}=0$ ) represents a population without cockroach migration from other neighbourhoods nor any reinfestation of cleaned households. The second case $\left(\Lambda=0\right.$ and $\left.\beta_{2} \neq 0\right)$ signifies a population absent of cockroach migration from other neighbourhoods as well, but in this case, reinfestation of cleaned households will be considered. The third and final case $\left(\Lambda \neq 0\right.$ and $\left.\beta_{2} \neq 0\right)$ represents a population containing both the influx of cockroaches from other neighbourhoods and reinfestation.

\subsection{Mathematical analysis of the household model}

In this section, we mathematically analyse Cases I and II. Due to the complexity of analytical computation, Case III will be analysed numerically in Section 3.2.

\subsubsection{Cockroach free equilibrium and derivation of $\boldsymbol{R}_{\mathbf{0}}$}

Because the Cockroach Free Equilibrium (CFE) is the same for Case I and Case II, to simplify the computation of $R_{0}$, we take $\Lambda=0$ and $\beta_{1}=\beta_{2}$. For this, $\beta_{1}=\beta_{2}$ signifies an equal chance of cockroaches migrating from an infested house to susceptible or cleaned houses. With these modifications, System 1 becomes:

$$
\begin{aligned}
\frac{\mathrm{d} S}{\mathrm{~d} t} & =-\frac{S}{N}[\beta(\sigma A+I)]+\alpha A+C(\delta+\phi), \\
\frac{\mathrm{d} A}{\mathrm{~d} t} & =-\kappa A-\alpha A+\frac{S}{N}[\beta(\sigma A+I)], \\
\frac{\mathrm{d} I}{\mathrm{~d} t} & =-\alpha I+\kappa A+\frac{C}{N}[\beta(\sigma A+I)], \\
\frac{\mathrm{d} C}{\mathrm{~d} t} & =-(\delta+\phi) C-\frac{C}{N}[\beta(\sigma A+I)]+\alpha I
\end{aligned}
$$

with $N(t)=S(t)+A(t)+I(t)+C(t)$. CFE is given by:

$$
\text { CFE : }\left(S^{*}, A^{*}, I^{*}, C^{*}\right)=\left(N_{0}, 0,0,0\right) .
$$


Since the total population is constant, it suffices to consider a system of three equations where $S(t)=N_{0}-(A+I+C)(t)$. Making this substitution into System 2, letting $\mu=\delta+\phi$, and performing the stability analysis on CFE we arrive at: (see Appendix 1 for details)

$$
F>0 \Longleftrightarrow \alpha(\alpha+\kappa)>\alpha \beta \sigma+\beta \kappa \Longleftrightarrow \frac{\beta(\alpha \sigma+\kappa)}{\alpha(\alpha+\kappa)}<1
$$

where $F=\alpha(\alpha+\kappa)-\alpha \beta \sigma-\beta \kappa$.

Hence, we define

$$
R_{0}=\frac{\beta(\alpha \sigma+\kappa)}{\alpha(\alpha+\kappa)}=\frac{\beta \sigma}{\alpha+\kappa}+\frac{\beta}{\alpha}\left(\frac{\kappa}{\alpha+\kappa}\right)
$$

and if we let,

$$
R_{0}^{A}=\frac{\beta \sigma}{\alpha+\kappa} \quad \text { and } \quad R_{0}^{I}=\frac{\beta}{\alpha}\left(\frac{\kappa}{\alpha+\kappa}\right)
$$

then $R_{0}$ becomes

$$
R_{0}=R_{0}^{A}+R_{0}^{I}
$$

Theorem 2.1: If $R_{0}=R_{0}^{A}+R_{0}^{I}<1$, then the CFE is locally asymptotically stable.

Proof Sketch.: (See Appendix 1 for full proof)

To show local stability of the cockroach free equilibrium (CFE), we must prove that all eigenvalues of the Jacobian matrix evaluated at the CFE, $J_{(0,0,0)}$, have negative real parts.

$$
J_{(0,0,0)}=\left[\begin{array}{ccc}
\beta \sigma-(\alpha+\kappa) & \beta & 0 \\
\kappa & -\alpha & 0 \\
0 & \alpha & -\mu
\end{array}\right] .
$$

The characteristic equation of the matrix $J_{(0,0,0)}$ is as follows:

$$
(-\lambda-\mu)\left(\lambda^{2}+\lambda(2 \alpha+\kappa-\beta \sigma)+\alpha^{2}+\alpha \kappa-\beta \kappa+-\alpha \beta \sigma\right)=0 .
$$

Therefore, the eigenvalues of $J_{(0,0,0)}$ are given by $\lambda_{1}=-\mu$, and $\lambda_{2,3}=\frac{1}{2}[(2 \alpha+\kappa-\beta \sigma) \pm$ $\left.\sqrt{4 \beta \kappa+(\kappa-\beta \sigma)^{2}}\right]$ and are guaranteed to be negative when $R_{0}<1$. Thus, the cockroach free equilibrium is locally asymptotically stable when $R_{0}<1$.

2.2.1.1. Interpretation of $\boldsymbol{R}_{\mathbf{0}}$. The basic reproductive number for our model, $R_{0}$, is defined to be the average number of secondary cockroach infestations and subsequent asthma attacks produced by a single infested household in a completely susceptible neighbourhood.

The basic reproductive number, $R_{0}$, for our model is given by the expression:

$$
R_{0}=\frac{\beta(\kappa+\alpha \sigma)}{\alpha(\alpha+\kappa)}
$$

Thus, $R_{0}$ is the sum of two basic reproductive numbers; $R_{0}^{A}$, which is the average number of newly infested households produced by cockroach migration from asymptomatic 
infested homes and $R_{0}^{I}$ which represents the contribution to $R_{0}$ by symptomatic infested households. Thus, $R_{0}$ can be represented as

$$
R_{0}=R_{0}^{A}+R_{0}^{I}=\frac{\beta \sigma}{\alpha+\kappa}+\frac{\beta \kappa}{\alpha(\alpha+\kappa)}=\frac{\beta \sigma}{\alpha+\kappa}+\frac{\beta}{\alpha}\left(\frac{\kappa}{\alpha+\kappa}\right) ;
$$

$R_{0}^{A}$ is equal to the product of the reduced infestation rate $(\beta \sigma)$ of asymptomatic households multiplied by the average time that these households spend with both the presence of cockroaches and the absence of asthma symptoms $(1 /(\alpha+\kappa))$. On the other hand, $R_{0}^{I}$ is equal to the product of the infestation rate of symptomatic households $(\beta)$ multiplied by the proportion of asymptomatic homes that move on to become symptomatic $(\kappa /(\alpha+\kappa))$ and the average time that household shows asthma symptoms and remains infested $(1 / \alpha)$.

To further study the model, we will calculate endemic equilibria for Cases I and II.

\subsubsection{Case I: $\Lambda=0$ and $\beta_{2}=0$}

The assumptions $\Lambda=0$ and $\beta_{2}=0$ lead to a special case of the household model. Biologically, $\Lambda=0$ implies that there is no influx of outside cockroaches into susceptible or clean houses. Also in this case, $\beta_{2}=0$ and no longer depends on $\beta_{1}$, which means that there is no possibility of a cleaned house becoming reinfested with cockroaches from other infested houses within the neighbourhood.

The cockroaches and asthma equilibrium $E^{*}$ is given in terms of $R_{0}$ by:

$$
\begin{aligned}
E^{*}= & \left(\frac{N_{0}}{R_{0}}, \frac{N_{0} \alpha \mu}{\kappa \mu+\alpha \mu+\kappa \alpha}\left(1-\frac{1}{R_{0}}\right), \frac{N_{0} \kappa \mu}{\kappa \mu+\alpha \mu+\kappa \alpha}\left(1-\frac{1}{R_{0}}\right),\right. \\
& \left.\frac{N_{0} \kappa \alpha}{\kappa \mu+\alpha \mu+\kappa \alpha}\left(1-\frac{1}{R_{0}}\right)\right)
\end{aligned}
$$

Thus, $E^{*}$ exists provided that $R_{0}>1$.

The characteristic polynomial of the Jacobian matrix evaluated at $E^{*}$ was calculated and found to be of the form $P(\lambda)=\lambda^{3}+a_{2} \lambda^{2}+a_{1} \lambda+a_{0}$. Because $a_{0}, a_{2}>0$ and $a_{2} a_{1}>a_{0}$, the conditions of the Routh-Hurwitz stability criterion (Hurwitz, 1964; Routh, 1877) were satisfied for the cockroaches and asthma equilibrium $\left(E^{*}\right)$.

Thus, the endemic equilibrium is locally stable if $R_{0}>1$.

Theorem 2.2: If $R_{0}=R_{0}^{A}+R_{0}^{I}>1$, then there exists an endemic equilibrium and it is locally asymptotically stable.

Proof Sketch.: (See Appendix 2 for full proof.)

To prove the stability of the endemic equilibrium when $R_{0}<1$, we first find the Jacobian matrix evaluated at $E^{*}$ and its corresponding characteristic polynomial. Let $R_{0}>1$ and

$$
A=\frac{\alpha \mu\left(R_{0}-1\right)}{\kappa \mu+\alpha \mu+\kappa \alpha} .
$$

Then the characteristic polynomial can be represented as $P(\lambda)=\lambda^{3}+a_{2} \lambda^{2}+a_{1} \lambda+a_{0}$ where

$$
a_{2}=(\alpha+\mu)+(\alpha+\kappa)\left[\frac{\kappa}{\alpha \sigma+\kappa}+A\right]
$$




$$
\begin{aligned}
& a_{1}=(\alpha+\kappa)(\alpha+\mu)\left[\frac{\kappa}{\alpha \sigma+\kappa}+A\right]+\alpha \mu-\kappa(\alpha+\kappa)\left[\frac{\alpha}{\alpha \sigma+\kappa}-A\right] ; \quad \text { and } \\
& a_{0}=\alpha \mu(\alpha+\kappa)\left[\frac{\kappa}{\alpha \sigma+\kappa}+A\right]+\alpha \kappa(\alpha+\kappa) A-\kappa \mu(\alpha+\kappa)\left[\frac{\alpha}{\alpha \sigma+\kappa}-A\right]
\end{aligned}
$$

In this case, $a_{0}, a_{2}>0$ and $a_{2} a_{1}>a_{0}$, thus the conditions of the Routh-Hurwitz stability criterion are met and the endemic equilibrium $E^{*}$ is locally asymptotically stable when $R_{0}>1$.

\subsubsection{Case II: $\Lambda=0$ and $\beta_{2} \neq 0$}

In this case, the assumption that $\Lambda=0$ and $\beta_{2} \neq 0$ signifies continued absence of the migration of cockroaches from outside the neighbourhood. However, the possibility of a household becoming reinfested after extermination is now being considered.

The system of equations produced by making these assumptions is as follows:

$$
\begin{aligned}
\frac{\mathrm{d} S}{\mathrm{~d} t} & =-\frac{S}{N_{0}}\left[\beta_{1}(\sigma A+I)\right]+\alpha A+\mu C, \\
\frac{\mathrm{d} A}{\mathrm{~d} t} & =-\kappa A-\alpha A+\frac{S}{N_{0}}\left[\beta_{1}(\sigma A+I)\right], \\
\frac{\mathrm{d} I}{\mathrm{~d} t} & =-\alpha I+\kappa A+\frac{C}{N_{0}}\left[\beta_{2}(\sigma A+I)\right], \\
\frac{\mathrm{d} C}{\mathrm{~d} t} & =-\mu C+\alpha I-\frac{C}{N_{0}}\left[\beta_{2}(\sigma A+I)\right] .
\end{aligned}
$$

By setting the right hand side of all equations in System (5) equal to zero and solving, we determined that the CFE and $R_{0}$ are equal to those in the previous case. We were also able to find equations for the endemic equilibrium in terms of the asymptomatic households:

$$
\begin{aligned}
S\left(A^{*}\right) & =\frac{N_{0}(\kappa+\alpha)}{\beta_{1}\left(\sigma+\frac{\kappa\left(\mu N+\beta_{2} \sigma A^{*}\right)}{\mu \alpha N-\kappa \beta_{2} A^{*}}\right)}, \quad I\left(A^{*}\right)=\frac{A^{*} \kappa\left(\mu N_{0}+\beta_{2} \sigma A^{*}\right)}{\mu \alpha N_{0}-\beta_{2} \kappa A^{*}} \quad \text { and } \\
C\left(A^{*}\right) & =\frac{\kappa}{\mu} A^{*} .
\end{aligned}
$$

Rewriting $S\left(A^{*}\right)$ in terms of $R_{0}$ gives:

$$
S\left(A^{*}\right)=\frac{N_{0}(\kappa+\alpha)}{\beta_{1}\left(\sigma+\frac{\kappa\left(\mu N+\beta_{2} \sigma A^{*}\right)}{\mu \alpha N-\kappa \beta_{2} A^{*}}\right)}=\frac{1}{R_{0}}\left(N_{0}-\frac{\beta_{2} \kappa}{\mu \alpha}\right) A^{*} .
$$

Thus, $S\left(A^{*}\right)$ exists as long as,

$$
N_{0}>\frac{\kappa}{\alpha} \cdot \frac{\beta_{2}}{\mu} .
$$

Therefore, $E^{*}$ exists, if the initial population is greater than the product of the rate at which a house becomes asthmatic times the average time a household waits before extermination and the rate at which a cleaned house becomes asthmatic multiplied by the average time a household waits before allergen removal. 
Table 3. Table of estimated values for all parameters.

\begin{tabular}{|c|c|c|c|}
\hline Parameter & Definition & Estimated Value & Reference \\
\hline$\Lambda$ & Influx rate of cockroaches from outside neighbourhoods & Varied & - \\
\hline$\beta_{1}$ & Migration rate to susceptible houses & Varied & Cornwell (1968) \\
\hline$\beta_{2}$ & Migration rate to cleaned houses & Varied & Cornwell (1968) \\
\hline$\sigma$ & Reduction factor for asymptomatic infested homes & & - \\
\hline$\alpha$ & Extermination rate & $\frac{1}{2} \frac{1}{\text { months }}$ & Rabito et al. (2017) \\
\hline$\delta$ & Allergen removal rate & $\frac{1}{6} \frac{1}{\text { months }}$ & Eggleston et al. (1999) \\
\hline$\phi$ & Rate of households waiting for allergen to dissipate & $\frac{1}{8 \text { months }}$ & Rabito et al. (2017) \\
\hline$\kappa$ & Cockroach reproduction rate & $\frac{1}{0.6} \frac{1}{\text { months }}$ & $\begin{array}{l}\text { Cornwell (1968), Chad Gore } \\
\text { and Schal (2004) }\end{array}$ \\
\hline
\end{tabular}

Using the fact that our population is constant and substituting Equations (6) and (7) in

$$
0=N_{0}-S\left(A^{*}\right)-A^{*}-I\left(A^{*}\right)-C\left(A^{*}\right)
$$

we get a quadratic polynomial in terms of $A^{*}$,

$$
0=N_{0}-\left(\frac{N_{0}}{R_{0}}-\frac{\beta_{2} \kappa}{R_{0} \mu \alpha} A^{*}\right)-A^{*}-\left(\frac{\mu \kappa N_{0} A^{*}+\kappa \beta_{2} \sigma\left(A^{*}\right)^{2}}{\mu \alpha N_{0}-\kappa \beta_{2} A^{*}}\right)-\left(\frac{\kappa}{\mu} A^{*}\right)
$$

Solving for $A^{*}$ gives

$$
\begin{aligned}
0= & {\left[-\kappa^{2} \beta_{2}^{2}-R_{0} \alpha(\kappa+\mu(1-\sigma))\right]\left(A^{*}\right)^{2}-\left[N_{0} \alpha \mu\left(R_{0}-1\right) \kappa \beta_{2}-\beta_{2} \kappa+(\kappa+\mu) \alpha R_{0}\right.} \\
& \left.+R_{0} \mu \kappa\right] A^{*}+\left(R_{0}-1\right) \mu^{2} \alpha^{2} N^{2} .
\end{aligned}
$$

Setting $\tau_{1}=-\kappa^{2} \beta_{2}^{2}-R_{0} \alpha(\kappa+\mu(1-\sigma)), \tau_{2}=-N_{0} \alpha \mu\left(R_{0}-1\right) \kappa \beta_{2}+\beta_{2} \kappa-(\kappa+\mu)$ $\alpha R_{0}-R_{0} \mu \kappa$, and $\tau_{3}=\left(R_{0}-1\right) \mu^{2} \alpha^{2} N^{2}$ gives

$$
f(A)=\tau_{1} A^{2}+\tau_{2} A+\tau_{3}
$$

(See Appendix 3 for a detailed calculation of Equation (8)).

Solutions of $f(A)=0$ will yield the endemic equilibrium for the case $\Lambda=0$ and $\beta_{2} \neq 0$. Since it is difficult to study the solution of $f\left(A^{*}\right)=0$ analytically, we will do so numerically using parameter values found in the literature (see Table 3 for parameter estimates).

The equation $f(A)=0$ will provide either one or two roots for $A^{*}$, which implies that a bifurcation may be present.

In particular, for the given set of parameter values:

$$
\begin{aligned}
& \alpha=0.736, \quad \beta_{1}=0.89, \quad \beta_{2}=2, \quad \kappa=\frac{1}{0.6}, \\
& \mu=\frac{1}{6}+\frac{1}{8}, \quad \sigma=\frac{1}{13} \quad \text { and } \quad N_{0}=100,
\end{aligned}
$$

there exist two values of $A^{*}$, which correspond to the equilibria $E_{1}^{*}$ and $E_{2}^{*}$. The Jacobian matrix for System 5 evaluated at the equilibrium $E_{1}^{*}$ results in three negative and one positive eigenvalue, which implies that $E_{1}^{*}$ is locally asymptotically unstable for the set of parameters. However, the Jacobian matrix for System 5 evaluated at the equilibrium $E_{2}^{*}$ gives all negative eigenvalues, which implies the equilibrium point $E_{2}^{*}$ is locally asymptotically stable. 


\subsection{Parameter values}

From the literature we were able to obtain estimated values for parameters: $\alpha, \beta_{1}, \beta_{2}, \gamma, \kappa$, $\Lambda, \sigma$, and $\phi$. It has been determined that it takes a little over two months for a home to be completely cockroach free after extermination, thus the extermination rate $(\alpha)$ was set to $\frac{1}{2}$ month $^{-1}$ (Rabito, Carlson, He, Werthmann, \& Schal, 2017). Our estimate for $\delta$ was taken from a study by Eggleston et. al. in which they concluded that allergen levels will decrease by 95\% with sustained allergen removal every 6 months, thus, $\delta$ was set to $\frac{1}{6}$ month $^{-1}$.

The parameter $\kappa$ was found by combining information from multiple sources. Since $\kappa$ is defined to be the rate at which asymptomatic infested households become symptomatic, it was necessary for us to find the time it takes to build up $8 \mathrm{U} / \mathrm{g}$ of allergen after the arrival of a single cockroach. We began by assuming a mated female was the first to enter the home. Therefore, within 17 days, the nymphs would emerge from her ootheca and approximately 24 would mature to adulthood (Cornwell, 1968). Assuming 50\% are female, we find that each female will produce 12 other females. Furthermore, from a study by Chad Gore and Schal (2004), we found that each female German cockroach will excrete approximately 1037.5 $\mathrm{U}$ of Bla $\mathrm{g} 1$ in her feces each day. Joining this information with the reproduction rate of Blatella germanica, the assumption that female nymphs produce the same amount of allergen as female adults, the average amount of dust in a home per square foot, and the average square footage of a home, led us to the conclusion that $\kappa=\frac{1}{0.6} \mathrm{month}^{-1}$. From this conclusion, we determined that households in the asymptomatic infested class will typically only have 1 cockroach, while those in the symptomatic infested class will have at least 13. Thus, we set $\sigma$, the reduction factor of $A$, to be equal to $\frac{1}{13}$.

It has also been shown previously that German cockroaches are $83 \%$ more likely to harbour in places where a scent was left from another cockroach (Bassirpour \& Zoratti, 2014; Cornwell, 1968). Therefore, the migration rate to a cleaned house $\left(\beta_{2}\right)$ is assumed to be greater than the migration rate into a susceptible house $\left(\beta_{1}\right)$ because of the presence of cockroach allergens. However, since an exact rate cannot be found, the values for $\beta_{1}$ and $\beta_{2}$ will be varied assuming $\beta_{2}>\beta_{1}$. For similar reasons, the value for the influx rate of cockroaches from outside the neighbourhood $(\Lambda)$ will also be varied. Table 3 contains a summary of estimated values for all parameters, along with appropriate references.

\section{Results}

\subsection{Bifurcations}

Typically, in an epidemiological model, $R_{0}<1$ guarantees that the population reaches the disease free equilibrium, and when $R_{0}>1$, the disease persists. A similar scenario exists when the reinfestation of clean houses is not considered, $\beta_{2}=0$. That is, if $R_{0}<1$, the CFE will be locally asymptotically stable and, contrarily when $R_{0}>1$ the endemic equilibrium is born and is locally asymptotically stable.However, for the case where reinfestation of cleaned houses is considered ( $\beta_{2} \neq 0$ ), we will show numerically that decreasing $R_{0}$ below one is necessary, but not sufficient to guarantee the elimination of cockroaches and subsequent asthma symptoms from the neighbourhood. This scenario can be explained through a backward bifurcation. 


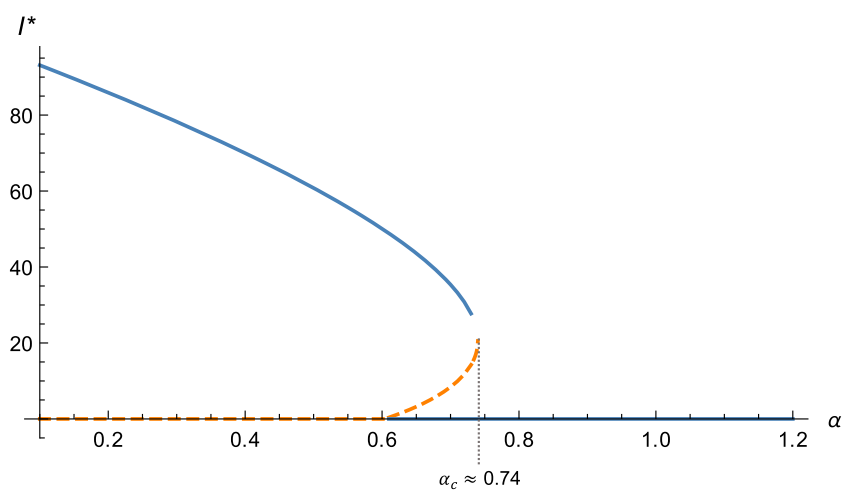

Figure 2. Subcritical backward bifurcation of $l^{*}$ with respect to migration rate $\alpha$ with parameter values: $\beta_{1}=0.80, \kappa=1 / 0.6, \beta_{2}=2, \phi=1 / 8$ and $\delta=1 / 6$. Stable equilibria are identified as solid blue lines, while unstable equilibria are represented with a dashed yellow line.

The backward bifurcation occurs when a small perturbation in parameter values results in either a change in the stability of the solutions or different solutions altogether. Since the polynomial in Equation (8) was not solved explicitly for $A^{*}$, there are no analytic conditions for backward bifurcations. Therefore, these bifurcations are given by solving the polynomial

$$
f(A)=\tau_{1} A^{2}+\tau_{2} A+\tau_{3}
$$

numerically for a given set of parameters (for Parameter values see Table 3 ). The polynomial was rewritten in terms of the infested households $(I)$ by using Equation (6) and plotted with respect to $\alpha$, which is shown to be the parameter to which $R_{0}$ is most sensitive (See Section 3.3). Supercritical backward bifurcation with respect to the migration rate $\beta_{1}$ can be found in Appendix 4.

\subsubsection{Subcritical backward bifurcation}

Figure 2 shows a subcritical backward bifurcation representing the relationship between infested asthmatic houses $I^{*}$ and the extermination rate $\alpha$. If $\alpha$ falls below the threshold value ( $\alpha=0.61$ ), then infestations and asthma symptoms are inevitable within the neighbourhood, irrespective of the initial population of symptomatic infested houses. This is due to the fact that the endemic equilibrium is the only stable equilibrium within this range of extermination rates. Similarly, because $I^{*}=0$ is the only stable equilibrium above the critical value $\left(\alpha_{c} \approx 0.74\right)$, then when $\alpha$ falls within this range, extermination alone will be sufficient for ridding the population of infestations and asthma symptoms. However, when the extermination rate is between the threshold value and the critical value, there are two possible outcomes, depending on the initial population of symptomatic infested houses. If the point of intersection between $\alpha$ and the initial value of $I$ lies below the line representing the unstable equilibrium, then the symptomatic infested class will go to zero. On the other hand, if this intersection lies above the unstable equilibrium, the presence of infestations and asthma symptoms will persist within the population. 


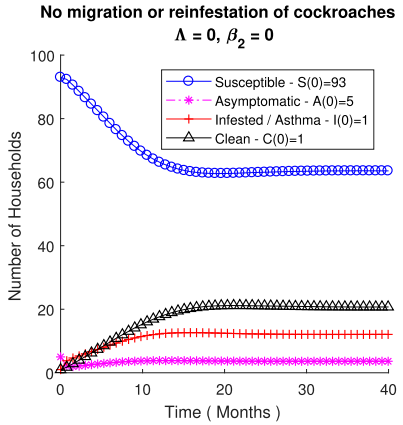

(a)

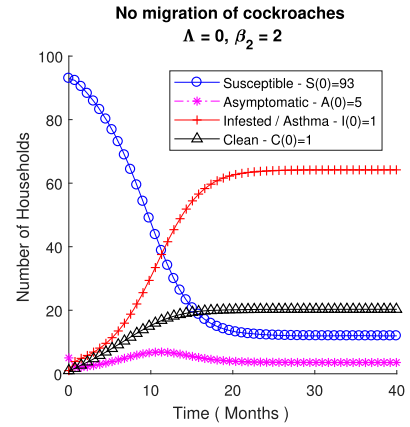

(b)

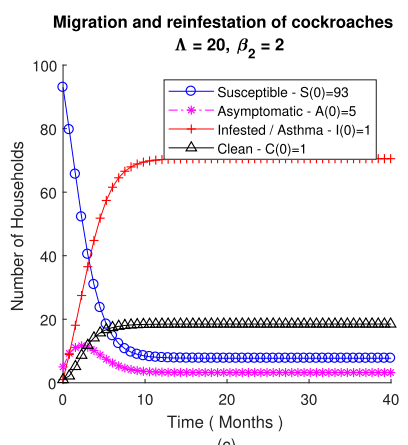

Figure 3. From left to right, numerical simulations of system behaviour for Case I, Case II and Case III. Simulations were performed using parameter estimates from Table 3 while varying values of $\Lambda$ and $\beta_{2}$.

\subsection{Numerical analysis}

Since the prevalence of asthma, in our model, is inextricably linked with the influx of cockroaches into a household, it is critical to understand if different routes of migration, namely from outside and within the neighbourhood, can lead to differing dynamics of the system. This is important in order to develop strategies that reduce the incidence of asthma symptoms triggered by cockroach allergens.

Numerical simulations were performed for the three different cases studied in Section 2.2 using the parameter estimations outlined in Table 3. The behaviour of the population constructed under these different scenarios is shown in Figure 3.

In every instance, the parameter values considered led to $R_{0}$ being greater than 1 , but the number of symptomatic infested households at equilibrium varies across cases. In case 1 , the population reached equilibrium after approximately 15 months, and approximately 18 months for case 2 . However, for case 2, there is an intersection of the susceptible and infested populations around the twelfth month. This is a critical point in which the number of infested houses exceeds the number of susceptible houses (Figure 3(b)).

The addition of the cockroach migration parameter in Case III $(\Lambda=20)$ appears to accelerate the time it takes for the system to reach equilibrium, as shown in Figure 3(c). Although the equilibrium values are similar compared to those in Case II, there is a slight increase in the value of the symptomatic infested households at equilibrium. This increase is related to the new influx of cockroaches, which extends the time a household is exposed to infestation and consequently develops asthma symptoms.

It is expected that when $R_{0}<1$, the population will be free of cockroaches and asthma symptoms. However, because a backward bifurcation is present in our model, even when this condition is fulfilled, if the reinfestation threshold $R_{\beta_{2}}$ is greater than one, it is still possible for the population to go to the endemic equilibrium (Figure 4). In Figure 5 a, we decrease the value of $R_{0}$ to 0.96 by modifying $\beta_{2}$, which also leads to $R_{\beta_{2}}=2.5$. In this case, $R_{0}<1$, but the population does not go to the cockroach free equilibrium. This implies that in order to prevent the occurrence of asthma symptoms related to the presence of cockroaches and its allergens, it is not only necessary to have $R_{0}<1$ but $R_{\beta_{2}}$ must also be less than one. To show this, $\beta_{2}$ was set to 0.7 , which resulted in the population going to the CFE, since $R_{0}<1$ and the value of $R_{\beta_{2}}$ decreased to 0.88 (Figure 5(b)). 


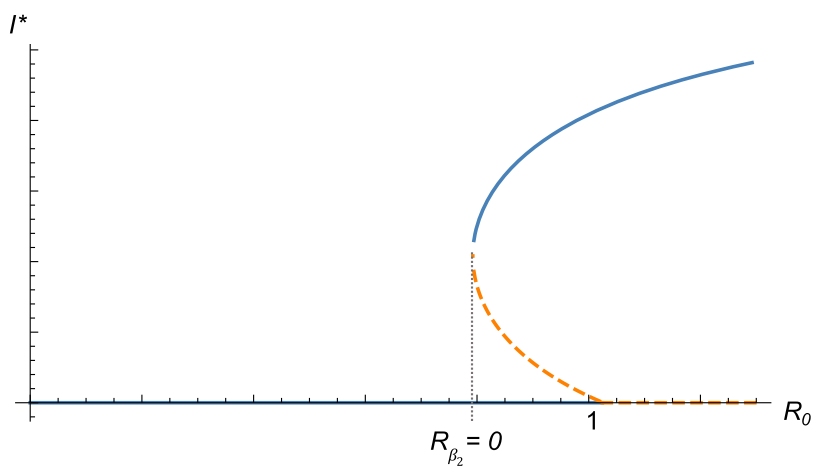

Figure 4. Graphic Sketch of the bifurcation and its relationship with $R_{0}$ and $R_{\beta_{2}}$.

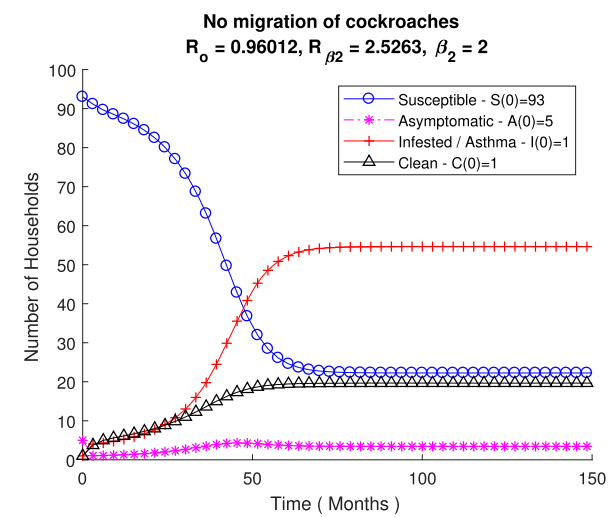

(a)

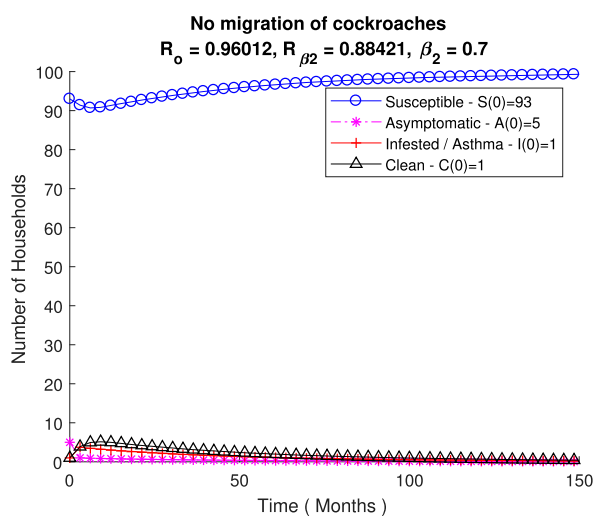

(b)

Figure 5. Effect of the change of $\beta_{2}$ on the equilibrium of System 1, (a) Case when $\beta_{2}=2$ and (b) Case when $\beta_{2}=0.7$. Simulations were performed using parameter estimates from Table 3 while varying values of $\beta_{2}$.

It is inferred from the analytical results that the presence of reinfestation $\left(\beta_{2}\right)$ in our model is at the root of this phenomenon. This parameter generates a new threshold condition analogous to the basic reproductive number:

$$
R_{\beta_{2}}=\beta_{2} \frac{1}{\alpha+(\delta+\phi)}
$$

$R_{\beta_{2}}$ is a product of the reinfestation rate of cockroaches in clean households $\beta_{2}$ and the average time a household remains clean $(1 /(\alpha+(\delta+\phi)))$. It explains how the clean households that become reinfested play a role in sustaining infestations in the neighbourhood. The importance of $R_{\beta_{2}}$ is highlighted when $R_{0}$ is less than one.

\subsubsection{Effect on parameter values}

The parameters which are believed to have the greatest effects on the model solutions and $R_{0}$ are the extermination $(\alpha)$, migration $\left(\beta_{1}\right)$ and reproduction $(\kappa)$ rates of cockroaches. In order to study how these parameters affect the recurrence of asthma symptoms in a 

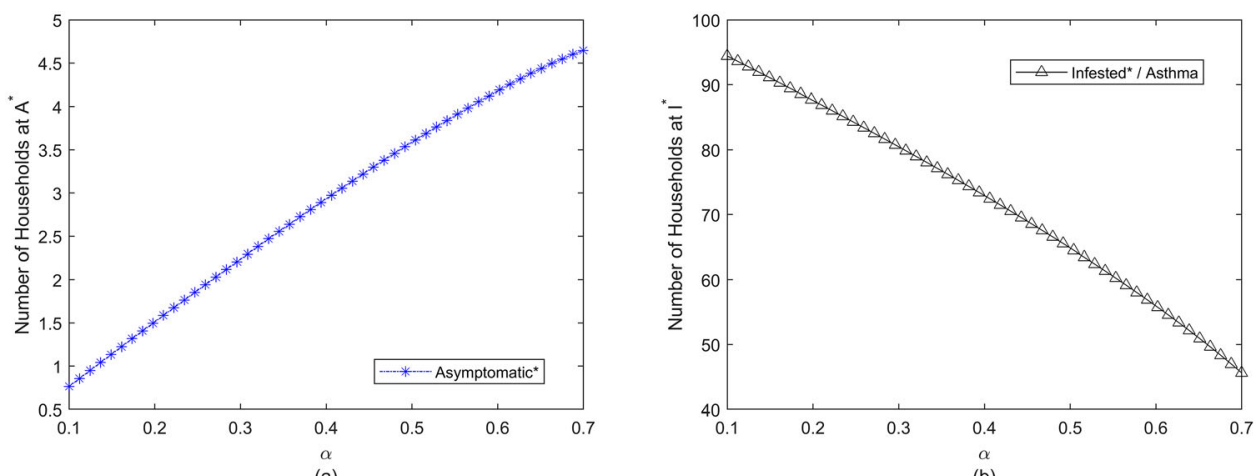

Figure 6. Effect of changes in extermination rate $\alpha$ on the equilibrium values of asymptomatic infested (a) and symptomatic infested households (b). Simulations were performed using parameter estimates from Table 3 while varying values of $\alpha$.

neighbourhood, the equilibrium solutions for the asymptomatic infested and symptomatic infested compartments were calculated as $\alpha, \kappa$ and $\beta_{1}$ varied.

In Figure 6, we see that there is a relatively linear relationship between $\alpha$ and the equilibrium value for asymptomatic and symptomatic infested houses. The correlation is positive for the asymptomatic infested households, and negative for the symptomatic households. This behaviour as $\alpha$ increases is attributable to the households spending more time in the asymptomatic infested class, evidenced by the increase of population at the equilibrium.

Common methods that prevent cockroach infestations such as vacuuming frequently, removing garbage around the home, and keeping food in places difficult for insects to access can decrease the reproduction rate of cockroaches $(\kappa)$. Since these activities limit the availability of resources, not performing them may increase $\kappa$. As a result, the number of households in the asymptomatic infested class will decline and shift more quickly to the symptomatic infested class (Figure 7(a)). Therefore the number of households in the symptomatic infested compartment at equilibrium will increase logarithmically with $\kappa$ (Figure 7(b)).

Similarly for $\beta_{1}$, as it increases, the equilibrium values of both asymptomatic and symptomatic infested households grow logaritmically (Figure 8). Due to the constant influx of cockroaches within the neighbourhood to the susceptible houses, the number of households in the $I$ compartment will continue to increase until it reaches a point of saturation where there are no longer any susceptible households.

\subsection{Sensitivity analysis}

Sensitivity analysis provides insight on how parameters affect the behaviour of a system. Finding the Normalized Sensitivity Index (NSI) aids in understanding the impact of changes in the parameters on a particular output. Forward Sensitivity Analysis (FSA) studies how a perturbation in the parameters can cause a perturbation change to a system (Cacuci, 2003; Griewank \& Walther, 2008). In our case local sensitivity analysis to the basic reproductive number (see Equation (3)) will be performed as well as to the model solutions with respect to time. To see the effect of each parameter on the reproduction number we 


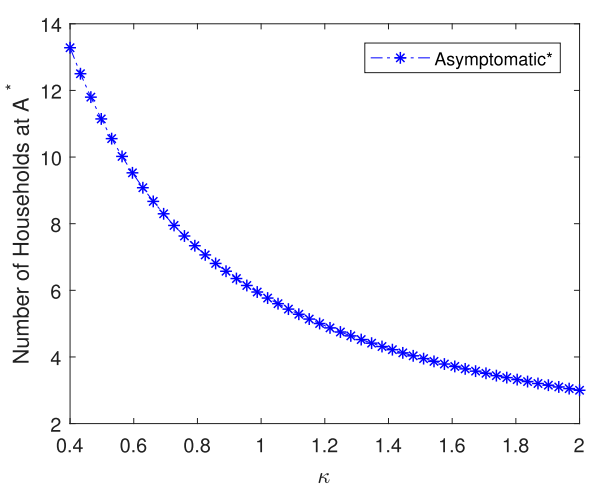

(a)

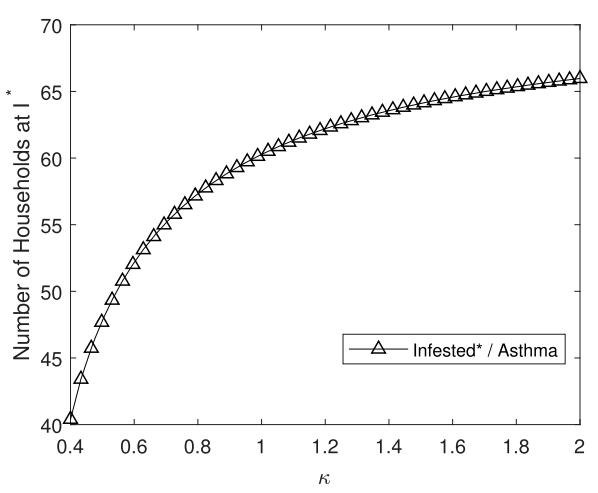

(b)

Figure 7. Effect of changes in reproduction rate $\kappa$ on the equilibrium values of asymptomatic infested (a) and symptomatic infested households (b). Simulations were performed using parameter estimates from Table 3 while varying values of $\kappa$.

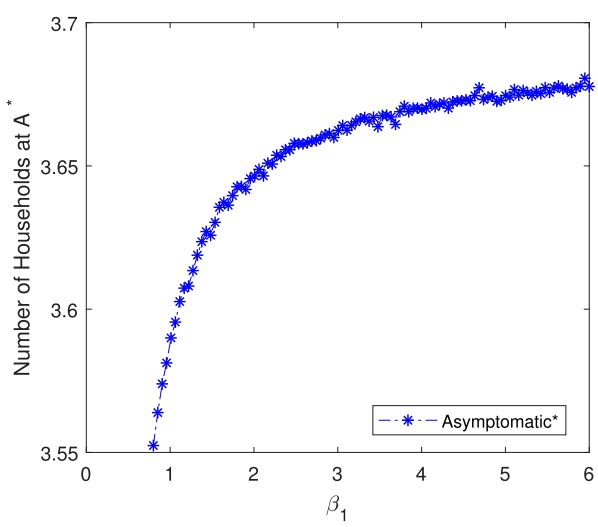

(a)

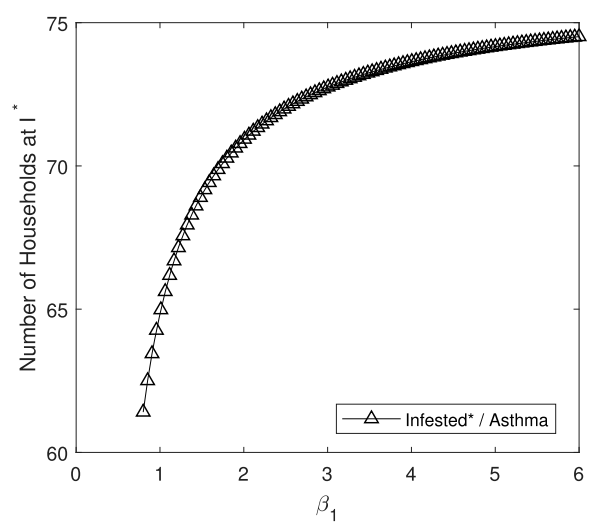

(b)

Figure 8. Effect of the parameter $\beta_{1}$ in the asymptomatic infested (a) and symptomatic infested households (b). Simulations were performed using parameter estimates from Table 3 while varying values of $\beta_{1}$.

calculated the sensitivity indices of $R_{0}$ with respect to the extermination rate $(\alpha)$, cockroach migration rate $\left(\beta_{1}\right)$, cockroach reproductive rate $(\kappa)$, and the reduction factor for asymptomatic infested households $(\sigma)$.

Let a parameter be represented as $p$, then the Normalized Sensitivity Index (NSI) is obtained by:

$$
S I=\lim _{\delta p \rightarrow 0} \frac{\frac{\delta R_{0}}{R_{0}}}{\frac{\delta p}{p}}=\frac{p}{R_{0}} \frac{\partial R_{0}}{\partial p} .
$$

Sensitivity indices were calculated using the estimated parameter values and can be found in Table 4. Sensitivity of the model solutions with respect to extermination rate $(\alpha)$ and cockroach reproductive rate $(\kappa)$ were also examined. Because these are parameters that can be controlled, this analysis was performed in order to determine the impact they have on household status over time. Details can be found in Appendix 5. 
Table 4. Normalized Sensitivity Indices.

\begin{tabular}{ccc}
\hline & Sensitivity Index & Value \\
\hline$S I_{\alpha}=\frac{\alpha}{R_{0}}\left(\frac{\partial R_{0}}{\partial \alpha}\right)$ & $S I_{\alpha}=-\frac{\alpha}{\alpha+\kappa}-\frac{\kappa}{\kappa+\alpha \sigma}$ & -1.20821 \\
$S I_{\beta}=\frac{\beta_{1}}{R_{0}}\left(\frac{\partial R_{0}}{\partial \beta_{1}}\right)$ & $S I_{\beta}=1$ & 1 \\
$S I_{\kappa}=\frac{\kappa}{R_{0}}\left(\frac{\partial R_{0}}{\partial \kappa}\right)$ & $S I_{\kappa}=\kappa\left(-\frac{1}{\alpha+\kappa}+\frac{1}{\kappa+\alpha \sigma}\right)$ & 0.20821 \\
$S I_{\sigma}=\frac{\sigma}{R_{0}}\left(\frac{\partial R_{0}}{\partial \sigma}\right)$ & $S I_{\sigma}=\frac{\alpha \sigma}{\kappa+\alpha \sigma}$ & 0.02256 \\
\hline
\end{tabular}

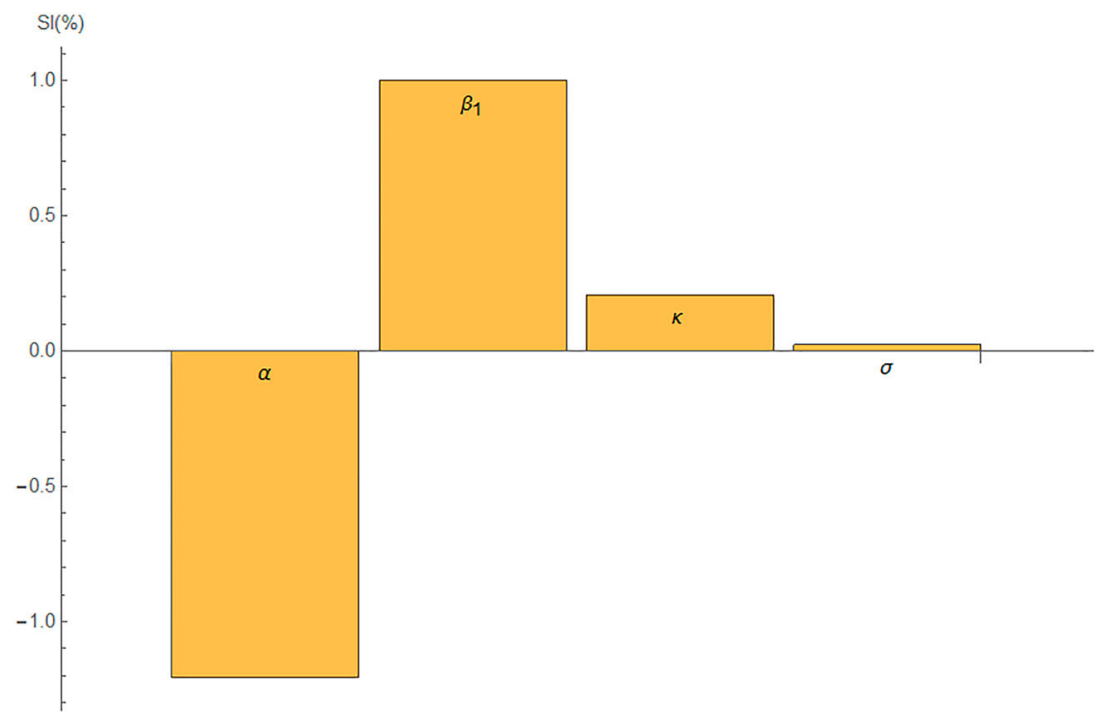

Figure 9. Normalized Sensitivity Index of $R_{0}$ parameters.

\subsubsection{Sensitivity analysis of $\boldsymbol{R}_{\mathbf{0}}$}

Considering the case when cockroach migration to clean households is zero $\left(\beta_{2}=0\right), R_{0}$ is most sensitive to the extermination rate $\alpha$, whose NSI was found to be -1.21 (Figure 9). Therefore, if $\alpha$ is increased by $1 \%$, the reproductive number will decrease by $1.21 \%$. Using the estimated parameter values, the reproductive number is equal to 1.574 . However, increasing $\alpha$ - a parameter that is easily controlled - by $30 \%$ will result in $R_{0}<1$. Hence, one would need to clean at least every six weeks in order to prevent cockroach infestations and any subsequent asthma symptoms.

\subsubsection{Sensitivity analysis to solutions}

Forward Sensitivity Analysis (FSA) with respect to $\Lambda, \kappa, \alpha, \delta$, and $\sigma$ was performed to determine the sensitivity of the model solutions to these parameters. We did not consider the sensitivity of each solution with respect to $\beta_{1}, \beta_{2}$, and $\phi$ because these parameters cannot be directly controlled. We saw that, among the controllable parameters, $\alpha$ and $\kappa$ have the largest impact on the basic reproductive number, so their relationship with the 


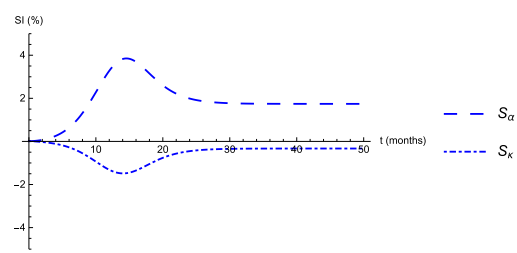

(a)

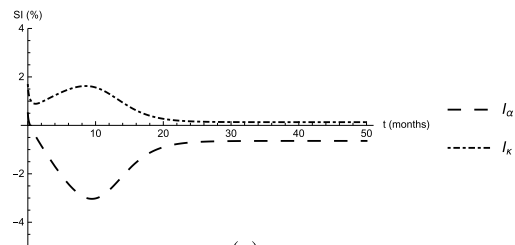

(c)

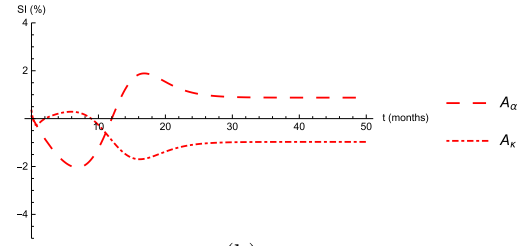

(b)

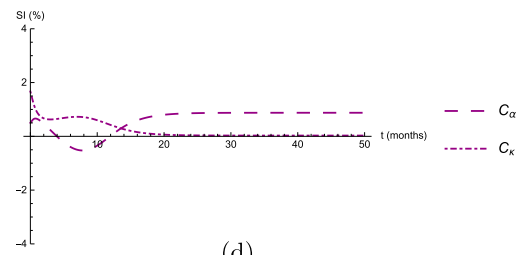

(d)

Figure 10. Normalized Sensitivity Index (NSI) over time of susceptible, asymptomatic infested, symptomatic infested, and clean households with respect to extermination rate and cockroach reproduction rate. (a) Change in SI of Susceptible Households. (b) Change in SI of Asymptomatic Infested Households. (c) Change in SI of Symptomatic Infested Households. (d) Change in SI of Clean Households.

model solutions are highlighted in Figure 10. The sensitivity over time of the susceptible class is shown with respect to $\alpha$ and $\kappa$ in Figure 10 a. The greatest impact to the susceptible households happens around 14 months, where the NSI with respect to $\alpha$ is approximately 4 . This increases the likelihood of staying as a susceptible household when periodical extermination is necessary. Contrarily, the NSI with respect to $\kappa$ is approximately -1.5 , which increases the possibility of changing states to an asymptomatic infested household. The opposite occurs in the sensitivity over time for the symptomatic infested class (Figure 10(c)). In this case, $\alpha$ peaks at around 1.5, and $\kappa$ has its peak at approximately -3 , which both occur after 10 months.

The sensitivity of asymptomatic infested households is shown with respect to $\alpha$ and $\kappa$ in Figure 10(b). This result is particularly interesting due to the shift from negative to a positive NSI for $\alpha$, and vice versa for $\kappa$, at approximately 10 months. In addition to this, there is also an intersection between the lines representing the sensitivity indices for $\kappa$ and $\alpha$ at around 11 months. A similar scenario exists, to a lesser extent, within the cleaned household compartment (Figure 10(d)). Although the intersection happens at roughly the same time, in this case, the NSI for both parameters remain positive.

\section{Discussion}

The increase in the prevalence of respiratory diseases such as asthma in recent years has led to an urgent need to identify better prevention or control methods for the most common triggers. Previous studies have shown that cockroaches and their allergens may be related to the development of asthma in both children and adults (Arruda et al., 2001; Cohn, Arbes, Jaramillo, Reid, \& Zeldin, 2006; Eggleston et al., 1999; Kang, 1976). Although there is no official cure for asthma, reduced exposure to these allergens may result in a decreased probability of having an asthma attack. To provide us with a better understanding of the relationship between cockroach infestations and the occurrence of asthma symptoms, a 
compartmental model that tracks the status of a household in response to the presence of cockroaches was developed.

In our model, the rates at which households exterminate cockroaches and remove the accompanying allergens were considered in order to determine the impact of cockroaches in triggering asthmatic symptoms. While studying this model, two different cases were considered. Firstly, a mathematical analysis of the case with exclusion of cockroach migration from other neighbourhoods $(\Lambda=0)$. This case was further divided into two sub cases: no reinfestation of cockroaches $\left(\beta_{2}=0\right)$ or reinfestation of cockroaches $\left(\beta_{2} \neq 0\right)$. The second case, which included the migration of cockroaches from other neighbourhoods $(\Lambda \neq 0)$, was only analysed numerically.

From the mathematical analysis of this model, in the case where $\Lambda=0$, a cockroach free equilibrium (CFE) for both sub-cases was found and $R_{0}$, the average number of secondary infestations produced by the presence of a single infestation in a population of households without cockroaches and asthma was also calculated. Moreover, CFE was shown to be locally asymptotically stable for both sub-cases when $R_{0}<1$. That is, there are no cockroaches and no asthma symptoms if $R_{0}<1$. The first sub case $\Lambda=0$ and $\beta_{2}=0$ was further studied in order to find the endemic equilibrium. This equilibrium was found to be locally asymptotically stable if $R_{0}>1$, i.e. the presence of cockroaches and asthma symptoms will persist in the population.

The second sub-case $\Lambda=0$ and $\beta_{2} \neq 0$ was solved numerically. As this case considers the reinfestation of clean households, a new threshold $R_{\beta_{2}}$ was established. This new reinfestation threshold is a direct consequence of households in the cleaned stage having the ability to return to the symptomatic infested class. From this, we conclude that $R_{0}<1$ is necessary, but not sufficient to guarantee the population reaches the cockroach free equilibrium; $R_{\beta_{2}}$ must also be less than 1 . This is attributable to the existence of a backward bifurcation in our system with respect to the parameters $\alpha$ and $\beta_{1}$. Mathematically, the presence of the backward bifurcation represents a bi-stability of the system due to the existence of two attractors when $R_{0}<1$, and once the initial conditions are modified it is possible to reach different equilibria. Biologically, this bifurcation signifies that it is more difficult to eliminate cockroaches in a neighbourhood than it is to prevent them since the effort needed to return to a cockroach free equilibrium post-infestation is larger than what is needed for prevention. In particular, with the set of parameters used, extermination every four weeks will enable the population to go to the CFE and eliminate cockroaches from the neighbourhood. However, extermination is needed every two weeks to guarantee that the symptomatic infested class will go to zero, independent of initial conditions.

From numerical and sensitivity analyses, it was determined that the controllable parameters which have the greatest impact on the reproduction number are the extermination rate $(\alpha)$ and cockroach reproductive rate $(\kappa)$. As a result, a decrease of $R_{0}$ below 1 is can be achieved by manipulating either of these parameters, but adjustment of $\alpha$ is the most effective. This is evident from its linear relationship with the equilibrium value of asymptomatic and symptomatic households, as well as its normalized sensitivity index. However, from examining sensitivity over time, it is clear that although $\alpha$ and $\kappa$ are useful for modifying $R_{0}$ and the equilibrium values, their effectiveness varies (Figure 10). Specifically, for symptomatic infested households, $\alpha$ has the greatest impact at approximately 10 months. It is also important to note that in the asymptomatic infested class, with regards to $\alpha$ there is a shift in the NSI from negative to positive at approximately 11 months. Furthermore, 
there is opposing shift in the influence of $\kappa$ from positive to negative at around 10 months. This phenomenon is representative of the changing impact of these parameters over time. Therefore, it is most efficient to manipulate the extermination rate before 10 months have passed, as that is when $\alpha$ will have the most influence on the system.

\section{Conclusion}

The results of mathematical analysis, numerical simulations, and sensitivity analysis imply that it is more effective to prevent infestation than to attempt to remove cockroaches once they have infested a house. Additionally, it was shown that when reinfestation is considered, it may not be enough to increase the extermination rate; but it is also necessary to increase the rate of allergen removal, as it is possible for reinfestations to sustain the presence of cockroaches. For future work, it is important to consider households of different socioeconomic statuses, in addition to the cost efficiency of extermination and allergen removal. Since cockroach infestations mainly affects lower-income households, treatments for them, as well as those for asthma symptoms are usually difficult to afford, having negative effects that should also be taken into account.

\section{Acknowledgments}

Authors want to give special thanks to Dr. Carlos Castillo-Chavez, Dr. Anuj Mubayi, and Dr. Leon Arriola, as well as Dr. Baltazar Cortes and Carlos Cruz for all of their help while the research was being conducted. We would also like to thank Dr. Maureen Rush for her assistance in editing the manuscript. This research was carried out at the Mathematical and Theoretical Biology Institute (MTBI), a summer program at the Simon A. Levin Mathematical, Computational and Modeling Sciences Center at Arizona State University (ASU).

\section{Disclosure statement}

No potential conflict of interest was reported by the authors.

\section{Funding}

This project has been partially supported by grants from the National Science Foundation (DMS1263374), the National Security Agency (H98230-15-1-0021), the Office of the President of ASU, and the Office of the Provost at ASU.

\section{References}

Arruda, L. K., Vailes, L. D., Ferriani, V. P., Santos, A. B. R., Pomés, A., \& Chapman, M. D. (2001). Cockroach allergens and asthma. Journal of Allergy and Clinical Immunology, 107(3), 419-428.

Bassirpour, G., \& Zoratti, E. (2014, December). Cockroach allergy and allergen-specific immunotherapy in asthma: Potential and pitfalls. Current Opinion in Allergy and Clinical Immunology, 14(6), 535-541.

Braman, S. S. (2006). The global burden of asthma. Chest, 130(1, Supplement), 4S-12S. Retrieved from http://www.sciencedirect.com/science/article/pii/S0012369215329524

Cacuci, D. G. (2003). Sensitivity \& uncertainty analysis. Volume 1: Theory. New York: Chapman and Hall/CRC.

Call, R. S., Smith, T. F., Morris, E., Chapman, M. D., \& Platts-Mills, T. A. (1992). Risk factors for asthma in inner city children. The Journal of Pediatrics, 121(6), 862-866. 
Chad Gore, J., \& Schal, C. (2004). Gene Expression and Tissue Distribution of the Major Human Allergen Bla g 1 in the German Cockroach, Blattella germanica L. (Dictyoptera: Blattellidae). Journal of Medical Entomology, 41(5), 953-960.

Cohn, R. D., Arbes, S. J., Jaramillo, R., Reid, L. H., \& Zeldin, D. C. (2006). National prevalence and exposure risk for cockroach allergen in U.S. households. Environmental Health Perspectives, 114(4), 522-526.

Cornwell, P. (1968). Rentokil library: The Cockroach: A laboratory insect and an industrial pest. London: Hutchinson.

Eggleston, P. A., \& Arruda, L. K. (2001, March). Ecology and elimination of cockroaches and allergens in the home. Journal of Allergy and Clinical Immunology, 107(3), S422S429.

Eggleston, P. A., Rosenstreich, D., Lynn, H., Gergen, P., Baker, D., Kattan, M., Mortimer, K., Mitchell, H., Ownby, D., \& Slavin, R. (1998, October). Relationship of indoor allergen exposure to skin test sensitivity in inner-city children with asthma. Journal of Allergy and Clinical Immunology, 102(4), 563-570.

Eggleston, P. A., Wood, R. A., Rand, C., Nixon, W. J., Chen, P. H., \& Lukk, P. (1999, October). Removal of cockroach allergen from inner-city homes. Journal of Allergy and Clinical Immunology, 104(4), 842-846.

Gelber, L. E., Seltzer, L. H., Bouzoukis, J. K., Pollart, S. M., Chapman, M. D., \& Platts-Mills, T. (1993). Sensitization and exposure to indoor allergens as risk factors for asthma among patients presenting to hospital. American Review of Respiratory Disease, 147, 573-578.

Griewank, A., \& Walther, A. (2008). Evaluating derivatives: principles and techniques of algorithmic differentiation. Vol. 105. Philadelphia, PA: Siam.

Hurwitz, A. (1964). On the conditions under which an equation has only roots with negative real parts. Selected papers on mathematical trends in control theory, 65, 273-284.

Kang, B. (1976). Study on cockroach antigen as a probable causative agent in bronchial asthma. The Journal of Allergy and Clinical Immunology, 58(3), 357-365.

Kermack, W. O., \& McKendrick, A. G. (1927). A contribution to the mathematical theory of epidemics. Proceedings of the royal society of london. Series A, Containing papers of a mathematical and physical character, 115(772), 700-721.

Ober, C., \& Yao, T. C. (2011, July). The genetics of asthma and allergic disease: A 21 st century perspective. Immunological Reviews, 242(1), 10-30.

Rabito, F. A., Carlson, J. C., He, H., Werthmann, D., \& Schal, C. (2017, January). A single intervention for cockroach control reduces cockroach exposure and asthma morbidity in children. Journal of Allergy and Clinical Immunology, 140(2), 565-570.

Rosenstreich, D. L., Eggleston, P., Kattan, M., Baker, D., Slavin, R. G., Gergen, P., Mitchell, H., McNiffMortimer, K., Lynn, H., Ownby, D., \& Malveaux, F. (1997, May). The role of cockroach allergy and exposure to cockroach allergen in causing morbidity among inner-city children with asthma. New England Journal of Medicine, 336(19), 1356-1363.

Routh, E. J. (1877). A treatise on the stability of a given state of motion: particularly steady motion. London: Macmillan and Company.

Rust, M. K., Owens, J. M., \& Reierson, D. A. (1995). Understanding and controlling the German cockroach. New York: Oxford University Press on Demand.

Sporik, R., Squillace, S. P., Ingram, J. M., Rakes, G., Honsinger, R. W., \& T. A. Platts-Mills (1999, August). Mite, cat, and cockroach exposure, allergen sensitisation, and asthma in children: A casecontrol study of three schools. Thorax, 54(8), 675-680.

Wang, C., Abou El-Nour, M. M., \& Bennett, G. W. (2008, February). Survey of pest infestation, asthma, and allergy in low-income housing. Journal of Community Health, 33(1), 31-39.

Wood, R. A., Eggleston, P. A., Rand, C., Nixon, W. J., \& Kanchanaraksa, S. (2001, July). Cockroach allergen abatement with extermination and sodium hypochlorite cleaning in inner-city homes. Annals of Allergy, Asthma and Immunology, 87(1), 60-64. 


\section{Appendix 1. Cockroach free equilibrium and $\boldsymbol{R}_{\mathbf{0}}$ derivation}

Making the substitution $S(t)=N_{0}-(A+I+C)(t)$ in (2) and letting $\mu=\delta+\phi$, the system of differential equations becomes

$$
\begin{aligned}
\frac{\mathrm{d} A}{\mathrm{~d} t} & =-\kappa A-\alpha A+\left(\frac{N_{0}-(A+I+C)}{N_{0}}\right) \beta_{1}(\sigma A+I), \\
\frac{\mathrm{d} I}{\mathrm{~d} t} & =-\alpha I+\kappa A+\frac{C \beta_{2}(\sigma A+I)}{N_{0}}, \\
\frac{\mathrm{d} C}{\mathrm{~d} t} & =-\mu C-\frac{C \beta_{2}(\sigma A+I)}{N_{0}}+\alpha I .
\end{aligned}
$$

The Jacobian matrix of the above system is:

$$
J=\left[\begin{array}{ccc}
-(\alpha+\kappa)+\beta_{1} \sigma\left(1-\frac{A+I+C}{N_{0}}\right) & \beta_{1}\left(1-\frac{A+I+C}{N_{0}}\right) & -\frac{\beta_{1}(I+\sigma A)}{N_{0}} \\
-\frac{\beta_{1}(I+\sigma A)}{N_{0}} & -\frac{\beta_{1}(I+\sigma A)}{N_{0}} & \frac{\beta_{2}(I+\sigma A)}{N_{0}} \\
\kappa+\frac{C \beta_{2} \sigma}{N_{0}} & -\alpha+\frac{C \beta_{2}}{N_{0}} & -\mu-\frac{\beta_{2}(I+\sigma A)}{N_{0}} \\
-\frac{C \beta_{2} \sigma}{N_{0}} & \alpha-\frac{C \beta_{2}}{N_{0}} & -c_{0}
\end{array}\right]
$$

To demonstrate local stability of the cockroach free equilibrium $\left(C F E:\left(S^{*}, A^{*}, I^{*}, C^{*}\right)=\right.$ $\left.\left(N_{0}, 0,0,0\right)\right)$, we must show that all the eigenvalues of the Jacobian matrix evaluated at the CFE have negative real parts.

The Jacobian matrix evaluated at the CFE is given by:

$$
J_{(0,0,0)}=\left[\begin{array}{ccc}
\beta \sigma-(\alpha+\kappa) & \beta & 0 \\
\kappa & -\alpha & 0 \\
0 & \alpha & -\mu
\end{array}\right] .
$$

The characteristic equation of the matrix $J_{(0,0,0)}$ is as follows:

$$
(-\lambda-\mu)\left(\lambda^{2}+\lambda(2 \alpha+\kappa-\beta \sigma)+\alpha^{2}+\alpha \kappa-\beta \kappa+-\alpha \beta \sigma\right)=0 .
$$

Therefore, the eigenvalues of $J_{(0,0,0)}$ are given by:

$$
\lambda_{1}=-\mu, \quad \text { and } \quad \lambda_{2,3}=\frac{1}{2}\left[(2 \alpha+\kappa-\beta \sigma) \pm \sqrt{4 \beta \kappa+(\kappa-\beta \sigma)^{2}}\right] .
$$

Since all parameters are assumed to be positive, $\lambda_{1}=-\mu$ is a negative eigenvalue.

We note that the discriminant is always positive. Therefore, both eigenvalues $\lambda_{2}$ and $\lambda_{3}$ are real for all values of the parameters. To further investigate the sign of the eigenvalues, we let:

$$
E=2 \alpha+\kappa-\beta \sigma \quad \text { and } F=\alpha(\alpha+\kappa)-\alpha \beta \sigma-\beta \kappa,
$$

then

$$
\lambda_{2,3}=\frac{1}{2}\left(-E \pm \sqrt{E^{2}-4 F}\right) .
$$

If $E>0$ and $F>0$, then $\sqrt{E^{2}-4 F}<E$ and the negativity of $\lambda_{2,3}$ is guaranteed.

$$
F>0 \Longleftrightarrow \alpha(\alpha+\kappa)>\alpha \beta \sigma+\beta \kappa \Longleftrightarrow \frac{\beta(\alpha \sigma+\kappa)}{\alpha(\alpha+\kappa)}<1 .
$$

Hence, we define

and if we let,

$$
R_{0}=\frac{\beta(\alpha \sigma+\kappa)}{\alpha(\alpha+\kappa)}=\frac{\beta \sigma}{\alpha+\kappa}+\frac{\beta}{\alpha}\left(\frac{\kappa}{\alpha+\kappa}\right)
$$

$$
R_{0}^{A}=\frac{\beta \sigma}{\alpha+\kappa} \quad \text { and } \quad R_{0}^{I}=\frac{\beta}{\alpha}\left(\frac{\kappa}{\alpha+\kappa}\right)
$$


then $R_{0}$ becomes

$$
R_{0}=R_{0}^{A}+R_{0}^{I}
$$

On the other hand $E>0$ is provided if

$$
2 \alpha+\kappa-\beta \sigma>0 \Longleftrightarrow \frac{\beta \sigma}{\alpha+\kappa}<1+\frac{\alpha}{\alpha+\kappa},
$$

that is by Equation (A2),

$$
R_{0}^{A}<1+\frac{\alpha}{\alpha+\kappa}
$$

Which is true since $R_{0}^{A}<1$. Therefore,

$$
F>0 \text { and } E>0 \Longleftrightarrow R_{0}<1,
$$

which means both eigenvalues $\lambda_{2}$ and $\lambda_{3}$ are negative.

All the eigenvalues of the $J_{(0,0,0)}$ are real and negative, thus the cockroach free equilibrium (CFE) is locally asymptotically stable.

\section{Appendix 2. Case I Endemic equilibrium proof}

In the case $\Lambda=0$ and $\beta 2=0$, the model becomes

$$
\begin{aligned}
\frac{\mathrm{d} A}{\mathrm{~d} t} & =-\kappa A-\alpha A+\frac{N-(A+I+C)}{N_{0}} \beta(\sigma A+I), \\
\frac{\mathrm{d} I}{\mathrm{~d} t} & =-\alpha I+\kappa A, \\
\frac{\mathrm{d} C}{\mathrm{~d} t} & =-\mu C+\alpha I,
\end{aligned}
$$

By setting the right hand side of the equations of the above system equal to zero, we found the cockroaches and asthma equilibrium $E^{*}$, which in terms of $R_{0}$ is given by:

$$
\begin{aligned}
E^{*}= & \left(\frac{N_{0}}{R_{0}}, \frac{N_{0} \alpha \mu}{\kappa \mu+\alpha \mu+\kappa \alpha}\left(1-\frac{1}{R_{0}}\right), \frac{N_{0} \kappa \mu}{\kappa \mu+\alpha \mu+\kappa \alpha}\left(1-\frac{1}{R_{0}}\right),\right. \\
& \left.\frac{N_{0} \kappa \alpha}{\kappa \mu+\alpha \mu+\kappa \alpha}\left(1-\frac{1}{R_{0}}\right)\right) .
\end{aligned}
$$

That is, $E^{*}$ exists provided that $R_{0}>1$.

The stability of the cockroach and asthma equilibrium will be shown by the Routh-Hurwitz Criterion. Following is the Jacobian matrix evaluated at $E^{*}$ :

$$
J_{\left(E^{*}\right)}=\left[\begin{array}{ccc}
\frac{\beta \sigma}{R_{0}}-\frac{\alpha \mu(\alpha+\kappa)}{\kappa \mu+\alpha \mu+\kappa \alpha} & \frac{\beta}{R_{0}}-\frac{\alpha \mu(\alpha+\kappa)}{\kappa \mu+\alpha \mu+\kappa \alpha} & -\frac{\alpha \mu(\alpha+\kappa)}{\kappa \mu+\alpha \mu+\kappa \alpha} \\
\left(R_{0}-1\right)-(\alpha+\kappa) & \left(R_{0}-1\right) & \left(R_{0}-1\right) \\
\kappa & -\alpha & 0 \\
0 & \alpha & -\mu
\end{array}\right]
$$

Let

$$
A=\frac{\alpha \mu\left(R_{0}-1\right)}{\kappa \mu+\alpha \mu+\kappa \alpha}>0 \quad \text { iff } \quad R_{0}>1,
$$

then, the Jacobian matrix $J_{\left(E^{*}\right)}$ can be reduced to:

$$
J_{\left(E^{*}\right)}=\left[\begin{array}{ccc}
-(\alpha+\kappa)\left[\frac{\kappa}{\alpha \sigma+\kappa}+A\right] & (\alpha+\kappa)\left[\frac{\alpha}{\alpha \sigma+\kappa}-A\right] & -(\alpha+\kappa) A \\
\kappa & -\alpha & 0 \\
0 & \alpha & -\mu
\end{array}\right] .
$$


The characteristic polynomial of $J_{\left(E^{*}\right)}$ will be given by solving:

$$
0=\left(J_{\left(E^{*}\right)}-\lambda I\right) \text {. }
$$

Recall, $E^{*}$ will be locally asymptotically stable if all eigenvalues $(\lambda)$ of the characteristic polynomial have negative real part. In this case, the characteristic polynomial is given by:

$$
\lambda^{3}+a_{2} \lambda^{2}+a_{1} \lambda+a_{0}=0
$$

where

$$
\begin{aligned}
a_{2} & =(\alpha+\mu)+(\alpha+\kappa)\left[\frac{\kappa}{\alpha \sigma+\kappa}+A\right] ; \\
a_{1} & =(\alpha+\kappa)(\alpha+\mu)\left[\frac{\kappa}{\alpha \sigma+\kappa}+A\right]+\alpha \mu-\kappa(\alpha+\kappa)\left[\frac{\alpha}{\alpha \sigma+\kappa}-A\right] ; \\
\text { and } \quad a_{0} & =\alpha \mu(\alpha+\kappa)\left[\frac{\kappa}{\alpha \sigma+\kappa}+A\right]+\alpha \kappa(\alpha+\kappa) A-\kappa \mu(\alpha+\kappa)\left[\frac{\alpha}{\alpha \sigma+\kappa}-A\right] .
\end{aligned}
$$

Therefore, $E^{*}$ will be locally asymptotically stable if $a_{0}>0, a_{2}>0$ and $a_{2} a_{1}>a_{0}$ (Routh - Hurwitz Sttability Criterion) (Hurwitz, 1964; Routh, 1877). The values of $a_{0}, a_{1}$ and $a_{2}$ can be further simplified as:

$$
\begin{aligned}
a_{2}= & \frac{1}{\alpha \sigma+\kappa}\{(\alpha+\mu)(\alpha \sigma+\kappa)+(\alpha+\kappa)[\kappa+A(\alpha \sigma+\kappa)]\}>0 \quad \text { iff } \quad\left(R_{0}>1\right) ; \\
a_{1}= & \frac{1}{\alpha \sigma+\kappa}\{\alpha \mu(\alpha \sigma+\kappa)+(\alpha+\kappa) \\
& \times[\kappa \mu+(\alpha \sigma+\kappa)(\alpha+\kappa+\mu) A]\}>0 \quad \text { iff } \quad\left(R_{0}>1\right) ;
\end{aligned}
$$

and $a_{0}=\alpha \mu(\alpha+\kappa)\left(R_{0}-1\right)>0$ iff $\left(R_{0}>1\right)$.

Notice that, $a_{0}$ and $a_{2}$ are positive iff $R_{0}>1$, thus if

$$
a_{2} a_{1}>a_{0} \Longleftrightarrow a_{2} a_{1}-a_{0}>0,
$$

then the local stability of $E^{*}$ is guaranteed.

Substituting the Equations (A6), (A7), and (A8) we can further simplify Equation (A9) and rewrite as:

$$
\begin{aligned}
a_{2} a_{1}-a_{0}= & (\alpha+\kappa)\left(\alpha^{2}+\mu \alpha+\mu^{2}\right) A+\frac{(\alpha+\mu)}{\alpha \sigma+\kappa}[\alpha \mu(\alpha \sigma+\kappa)+\kappa \mu(\alpha+\kappa)] \\
& +\left\{\frac{\kappa(\alpha+\kappa)}{\alpha \sigma+\kappa}(\alpha+\kappa) A\right\} \\
& \times\left\{\alpha \mu(\alpha \sigma+\kappa)+\frac{\kappa \mu(\alpha+\kappa)}{\alpha \sigma+\kappa} \kappa \mu(\alpha+\kappa)+(\alpha+\kappa)(\alpha+\kappa+\mu) A\right\} \\
> & 0
\end{aligned}
$$

By Routh Hurwitz Criterion, all the eigenvalues for the characteristic equation are negative or have negative real part since $a_{0}>0, a_{2}>0$ and $a_{2} a_{1}>a_{0}$.

\section{Appendix 3. Case II Endemic equilibrium proof}

In this appendix, the calculations to obtain $E^{*}$ where reinfestation is possible $(\lambda=0, \beta 2 \neq 0)$ are shown. By solving System 2 in terms of A, we obtain:

$$
S(A)=\frac{N_{0}(\kappa+\alpha)}{\beta_{1}\left(\sigma+\frac{\kappa\left(\mu N_{0}+\beta_{2} \sigma A\right)}{\mu \alpha N_{0}-\kappa \beta_{2} A}\right)}, I(A)=\frac{A \kappa\left(\mu N_{0}+\beta_{2} \sigma A\right)}{\mu \alpha N_{0}-\beta_{2} \kappa A} \quad \text { and } \quad C(A)=\frac{\kappa}{\mu} A
$$


Rewriting $S(A)$ in terms of $R_{0}$ gives:

$$
\begin{gathered}
S(A)=\frac{N_{0}(\kappa+\alpha)}{\beta_{1}\left(\sigma+\frac{\kappa\left(\mu N_{0}+\beta_{2} \sigma A\right)}{\mu \alpha N_{0}-\kappa \beta_{2} A}\right)}=\frac{1}{R_{0}}\left(N_{0}-\frac{\beta_{2} \kappa}{\mu \alpha}\right) A \\
0=N_{0}-(S+A+I+C)
\end{gathered}
$$

Substituting in our equations for $S(A), I(A)$, and $C(A)$ gives

$$
0=N_{0}-\frac{N_{0}}{R_{0}}+\frac{\beta_{2} \kappa A}{R_{0} \mu \alpha}-A-\frac{\mu N_{0} \kappa A+\kappa \beta_{2} \sigma A^{2}}{\mu \alpha N_{0}-\kappa \beta_{2} A}-\frac{\kappa}{\mu} A
$$

By setting $\mu \alpha N_{0}-\kappa \beta_{2} A=L$, we obtain:

$$
\begin{aligned}
& 0=N_{0} R_{0} \mu \alpha L-N_{0} \mu \alpha L+\beta_{2} \kappa L A-R_{0} \mu \alpha L A-R_{0} \mu \alpha\left(\mu N_{0} \kappa A+\kappa \beta_{2} \sigma A^{2}\right)-\kappa R_{0} \alpha L A \\
& 0=N_{0} \mu \alpha L\left(R_{0}-1\right)+\beta_{2} \kappa L A-R_{0} \alpha L A(\mu+\kappa)-R_{0} \mu \alpha \kappa A\left(\mu N_{0}+\beta_{2} \sigma A\right)
\end{aligned}
$$

Simplifying each section of the above equation gives

$$
\begin{aligned}
N_{0} \mu \alpha L\left(R_{0}-1\right) & =N_{0} \mu \alpha\left(\mu \alpha N_{0}-\kappa \beta_{2} A\right)\left(R_{0}-1\right) \\
& =\left(R_{0}-1\right)\left(\mu^{2} \alpha^{2} N_{0}^{2}-\kappa \beta_{2} N_{0} \mu \alpha A\right) \\
& =\left(R_{0}-1\right) \mu^{2} \alpha^{2} N_{0}^{2}-\left(R_{0}-1\right) \kappa \beta_{2} N_{0} \mu \alpha A \\
\beta_{2} \kappa L A & =\beta_{2} \kappa\left(\mu \alpha N_{0}-\kappa \beta_{2} A\right) A \\
& =\mu \alpha N_{0} \beta_{2} \kappa A-\kappa^{2} \beta_{2}^{2} A^{2} \\
-R_{0} \alpha L A(\mu+\kappa) & =-R_{0} \alpha\left(\mu \alpha N_{0}-\kappa \beta_{2} A\right) A(\kappa+\mu) \\
& =\left[-\mu \alpha^{2} N_{0} R_{0} A+\kappa \beta_{2} R_{0} \alpha A^{2}\right](\kappa+\mu) \\
& =-(\kappa+\mu) \mu \alpha^{2} N_{0} R_{0} A+(\kappa+\mu) \kappa \beta_{2} R_{0} \alpha A^{2} \\
-R_{0} \mu \alpha \kappa A\left(\mu N_{0}+\beta_{2} \sigma A\right) & =-R_{0} \mu^{2} \alpha \kappa N_{0} A-R_{0} \mu \alpha \kappa \beta_{2} \sigma A^{2}
\end{aligned}
$$

Plugging the simplified expressions back in to the original equation gives

$$
\begin{aligned}
0= & \left(R_{0}-1\right) \mu^{2} \alpha^{2} N_{0}^{2}-\left(R_{0}-1\right) \kappa \beta_{2} N_{0} \mu \alpha A+\mu \alpha N_{0} \beta_{2} \kappa A-\kappa^{2} \beta_{2}^{2} A^{2}-(\kappa+\mu) \mu \alpha^{2} N_{0} R_{0} A \\
& +(\kappa+\mu) \kappa \beta_{2} R_{0} \alpha A^{2}-R_{0} \mu^{2} \alpha \kappa N_{0} A-R_{0} \mu \alpha \kappa \beta_{2} \sigma A^{2} \\
0= & {\left[-\kappa^{2} \beta_{2}^{2}+(\kappa+\mu) \kappa \beta_{2} R_{0} \alpha-R_{0} \mu \alpha \kappa \beta_{2} \sigma\right] A^{2} } \\
& +\left[-\left(R_{0}-1\right) \kappa \beta_{2} N_{0} \mu \alpha+\mu \alpha N_{0} \beta_{2} \kappa-(\kappa+\mu) \mu \alpha^{2} N_{0} R_{0}-R_{0} \mu^{2} \alpha \kappa N_{0}\right] A \\
& +\left[\left(R_{0}-1\right) \mu^{2} \alpha^{2} N_{0}^{2}\right] \\
0= & \kappa \beta_{2}\left[-\kappa \beta_{2}+(\kappa+\mu) R_{0} \alpha-R_{0} \mu \alpha \sigma\right] A^{2} \\
& -N_{0} \alpha \mu\left[\left(R_{0}-1\right) \kappa \beta_{2}-\beta_{2} \kappa+(\kappa+\mu) \alpha R_{0}-R_{0} \mu \kappa\right] A \\
& +\left[\left(R_{0}-1\right) \mu^{2} \alpha^{2} N_{0}^{2}\right]
\end{aligned}
$$


The final polynomial in terms of $A$ then becomes

$$
\begin{aligned}
0= & -\kappa \beta_{2}\left[\kappa \beta_{2}-R_{0} \alpha(\kappa+\mu(1-\sigma))\right] A^{2}-N_{0} \alpha \mu\left[\left(R_{0}-1\right) \kappa \beta_{2}-\beta_{2} \kappa+(\kappa+\mu) \alpha R_{0}-R_{0} \mu \kappa\right] A \\
& +\left[\left(R_{0}-1\right) \mu^{2} \alpha^{2} N_{0}^{2}\right]
\end{aligned}
$$

By setting

$$
\begin{aligned}
& \tau_{1}=-\kappa \beta_{2}\left[\kappa \beta_{2}-R_{0} \alpha(\kappa+\mu(1-\sigma))\right] \\
& \tau_{2}=-N_{0} \alpha \mu\left[\left(R_{0}-1\right) \kappa \beta_{2}-\beta_{2} \kappa+(\kappa+\mu) \alpha R_{0}-R_{0} \mu \kappa\right] \\
& \tau_{3}=\left[\left(R_{0}-1\right) \mu^{2} \alpha^{2} N_{0}^{2}\right]
\end{aligned}
$$

We now have

$$
0=\tau_{1} A^{2}+\tau_{2} A+\tau_{3}
$$

\section{Appendix 4. Backward supercritical bifurcation}

The backward supercritical bifurcation shown in Figure A1 represents the relationship between the infested houses $\left(I^{*}\right)$ and the migration rate of cockroaches from infested to cleaned houses $\beta_{1}$. If $\beta_{1}$ falls below the critical value $\left(\beta_{1_{c}} \approx 0.7944\right)$, then the number of symptomatic infested households will always go to zero. This is due to the fact that the cockroach free equilibrium is locally asymptotically stable within this range. Similarly, because the endemic equilibrium is the only stable equilibrium above the threshold value $\left(\beta_{1} \approx 1.0244\right)$, when $\beta_{1}$ falls within this range, infestations and subsequent asthma symptoms will be inevitable within the population, independent of initial conditions. However, when the migration rate is between the threshold value and the critical value, there are two possible outcomes, depending on the initial population of symptomatic infested houses. If the point of intersection between $\beta_{1}$ and the initial value of $I$ lies below the line representing the unstable equilibrium, then the symptomatic infested class will go to zero. On the other hand, if this intersection lies above the unstable equilibrium, the presence of infestations and asthma symptoms will persist within the population. Additionally, the hysteresis effect is also present in this graph. That is, we need to maximize the migration rate of cockroaches in order to eventually return to the cockroach free equilibrium and eradicate asthma symptoms in a household.

Due to the fact that $\beta_{1}$ is not a parameter that is easily controlled, we mainly considered the the relationship of infested houses with the extermination rate, $\alpha$.

\section{Appendix 5. Sensitivity analysis}

To calculate the sensitivity index of the solutions we found the partial differential equations with respect to each controllable parameter that impacted $R_{0}(\kappa$ and $\alpha)$.

Forward Sensitivity Equations (FSE) with respect to $\alpha$ and $\kappa$ are as follows:

$$
\begin{aligned}
\frac{\mathrm{d}}{\mathrm{d} t}\left[\frac{\partial S}{\partial \alpha}\right]= & -\frac{\Lambda}{N}\left(\frac{\partial S}{\partial \alpha}\right)-\frac{\sigma \beta_{1}}{N}\left(\frac{\partial S}{\partial \alpha} A+\frac{\partial A}{\partial \alpha} S\right)-\frac{\beta_{1}}{N}\left(\frac{\partial S}{\partial \alpha} I+\frac{\partial I}{\partial \alpha} S\right)+\frac{\partial A}{\partial \alpha} \alpha+A \\
& +\frac{\partial C}{\partial \alpha} \delta+\frac{\partial C}{\partial \alpha} \phi \\
\frac{\mathrm{d}}{\mathrm{d} t}\left[\frac{\partial S}{\partial \kappa}\right]= & -\frac{\Lambda}{N}\left(\frac{\partial S}{\partial \kappa}\right)-\frac{\sigma \beta_{1}}{N}\left(\frac{\partial S}{\partial \kappa} A+\frac{\partial A}{\partial \kappa} S\right)-\frac{\beta_{1}}{N}\left(\frac{\partial S}{\partial \kappa} I+\frac{\partial I}{\partial \kappa} S\right)+\frac{\partial A}{\partial \kappa} \alpha+\frac{\partial C}{\partial \kappa} \delta+\frac{\partial C}{\partial \kappa} \phi \\
\frac{\mathrm{d}}{\mathrm{d} t}\left[\frac{\partial A}{\partial \alpha}\right]= & -\frac{\partial A}{\partial \alpha} \kappa-\frac{\partial A}{\partial \alpha} \alpha-A+\frac{\Lambda}{N}\left(\frac{\partial S}{\partial \alpha}\right)+\frac{\sigma \beta_{1}}{N}\left(\frac{\partial S}{\partial \alpha} A+\frac{\partial A}{\partial \alpha} S\right)+\frac{\beta_{1}}{N}\left(\frac{\partial S}{\partial \alpha} I+\frac{\partial I}{\partial \alpha} S\right) \\
\frac{\mathrm{d}}{\mathrm{d} t}\left[\frac{\partial A}{\partial \kappa}\right]= & -\frac{\partial A}{\partial \kappa} \kappa-A-\frac{\partial A}{\partial \kappa} \alpha+\frac{\Lambda}{N}\left(\frac{\partial S}{\partial \kappa}\right)+\frac{\sigma \beta_{1}}{N}\left(\frac{\partial S}{\partial \kappa} A+\frac{\partial A}{\partial \kappa} S\right)+\frac{\beta_{1}}{N}\left(\frac{\partial S}{\partial \kappa} I+\frac{\partial I}{\partial \kappa} S\right) \\
\frac{\mathrm{d}}{\mathrm{d} t}\left[\frac{\partial I}{\partial \alpha}\right]= & -\frac{\partial I}{\partial \alpha} \alpha-I+\frac{\partial A}{\partial \alpha} \kappa+\frac{\Lambda}{N}\left(\frac{\partial C}{\partial \alpha}\right)+\frac{\sigma \beta_{2}}{N}\left(\frac{\partial C}{\partial \alpha} A+\frac{\partial A}{\partial \alpha} C\right)+\frac{\beta_{2}}{N}\left(\frac{\partial C}{\partial \alpha} I+\frac{\partial I}{\partial \alpha} C\right)
\end{aligned}
$$




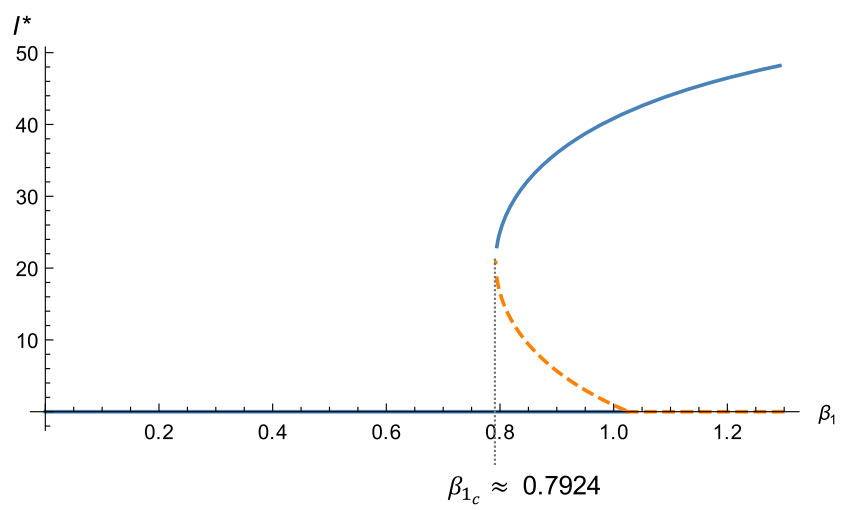

Figure A1. Supercritical backward bifurcation of $I^{*}$ with respect to migration rate $\beta_{1}$ with parameter values: $\alpha=0.736, \kappa=1 / 0.6, \beta_{2}=2, \phi=1 / 8$ and $\delta=1 / 6$. In this plot, blue lines represent stable equilibria and the unstable equilibrium is represented by the dashed yellow line.

$$
\begin{aligned}
\frac{\mathrm{d}}{\mathrm{d} t}\left[\frac{\partial I}{\partial \kappa}\right]= & -\frac{\partial I}{\partial \kappa} \alpha+\frac{\partial A}{\partial \kappa} \kappa+A+\frac{\Lambda}{N}\left(\frac{\partial C}{\partial \kappa}\right)+\frac{\sigma \beta_{2}}{N}\left(\frac{\partial C}{\partial \kappa} A+\frac{\partial A}{\partial \kappa} C\right)+\frac{\beta_{2}}{N}\left(\frac{\partial C}{\partial \kappa} I+\frac{\partial I}{\partial \kappa} C\right) \\
\frac{\mathrm{d}}{\mathrm{d} t}\left[\frac{\partial C}{\partial \alpha}\right]= & -\frac{\partial C}{\partial \alpha} \delta-\frac{\partial C}{\partial \alpha} \phi-\frac{\Lambda}{N}\left(\frac{\partial C}{\partial \alpha}\right)-\frac{\sigma \beta_{2}}{N}\left(\frac{\partial C}{\partial \alpha} A+\frac{\partial A}{\partial \alpha} C\right)-\frac{\beta_{2}}{N}\left(\frac{\partial C}{\partial \alpha} I+\frac{\partial I}{\partial \alpha} C\right) \\
& +\frac{\partial I}{\partial \alpha} \alpha+I \\
\frac{\mathrm{d}}{\mathrm{d} t}\left[\frac{\partial C}{\partial \kappa}\right]= & -\frac{\partial C}{\partial \kappa} \delta-\frac{\partial C}{\partial \kappa} \phi-\frac{\Lambda}{N}\left(\frac{\partial C}{\partial \kappa}\right)-\frac{\sigma \beta_{2}}{N}\left(\frac{\partial C}{\partial \kappa} A+\frac{\partial A}{\partial \kappa} C\right)-\frac{\beta_{2}}{N}\left(\frac{\partial C}{\partial \kappa} I+\frac{\partial I}{\partial \kappa} C\right)+\frac{\partial I}{\partial \kappa} \alpha
\end{aligned}
$$

